\title{
3D aerodynamic analysis and optimization of a gliding locust wing using Nash genetic algorithm
}

\author{
Hamid Isakhani * and Shigang Yue ${ }^{\dagger}$ \\ School of Computer Science, University of Lincoln, LN6 7TS, UK \\ Caihua Xiong ${ }^{\ddagger}$ and Wenbin Chen ${ }^{\S}$ \\ Huazhong University of Science and Technology, Wuhan, 430074, China
}

\begin{abstract}
Natural fliers glide and minimize wing articulation to conserve energy for endured and long range flights. Elucidating the underlying physiology of such capability could potentially address numerous challenging problems in flight engineering. This study investigates the aerodynamic characteristics of an insect species called desert locust (Schistocerca gregaria) with an extraordinary gliding skills at low Reynolds number. Here, locust tandem wings are subjected to a computational fluid dynamics (CFD) simulation using 2D and 3D Navier-Stokes equations revealing fore-hindwing interactions, and the influence of their corrugations on the aerodynamic performance. Furthermore, the obtained CFD results are mathematically parameterized using PARSEC method and optimized based on a novel fusion of Genetic Algorithms and Nash game theory to achieve Nash equilibrium being the optimized wings. It was concluded that the lift-drag (gliding) ratio of the optimized profiles were improved by at least $77 \%$ and $150 \%$ compared to the original wing and the published literature, respectively. Ultimately, the profiles are integrated and analyzed using 3D CFD simulations that demonstrated a 14\% performance improvement validating the proposed wing models for further fabrication and rapid prototyping presented in the future study.
\end{abstract}

\section{Nomenclature}

$\mathrm{b} \quad=$ Wingspan $(\mathrm{mm})$

c $\quad=$ Wing chord $(\mathrm{mm})$

$C_{D}=\frac{2 D}{\rho U^{2} S} \quad=\quad$ Drag coefficient

$C_{L}=\frac{2 L}{\rho U^{2} S} \quad=\quad$ Lift coefficient

$C_{P}=\frac{P-P_{\infty}}{0.5 \rho U^{2}}=$ Pressure coefficient

\footnotetext{
*Ph.D. Candidate, School of Computer Science, hamid.isakhani@gmail.com, AIAA Student Member.

${ }^{\dagger}$ Professor and Head of the Computational Intelligence Laboratory (CIL), School of Computer Science.

$\$$ Professor and Head of the State Key Laboratory of Digital Manufacturing Equipment and Technology.

$\S$ Associate Professor, The State Key Laboratory of Digital Manufacturing Equipment and Technology.
} 


$$
\begin{aligned}
& D \quad=\operatorname{Drag}(\mathrm{N}) \\
& f_{1}, f_{2} \quad=\text { Player objective functions } \\
& g \quad=\text { generation (an iteration in a GA optimization problem) } \\
& l \quad=\text { Length }(\mathrm{m}) \\
& L=\operatorname{Lift}(\mathrm{N}) \\
& N \quad=\text { Nash Equilibrium } \\
& p_{1}, p_{2} \quad=\text { Player strategies } \\
& P \quad=\text { Static pressure }\left(\mathrm{Nm}^{-2}\right) \\
& P_{\infty} \quad=\text { Freestream static pressure }\left(\mathrm{Nm}^{-2}\right) \\
& q=\frac{\rho U^{2} S}{2} \quad=\text { Dynamic pressure } \\
& R e=U l / v_{\infty}=\text { Reynolds number } \\
& S \quad=\text { Wing planform area }\left(\mathrm{m}^{2}\right) \\
& S t=f l / U=\text { Strouhal number }(f \text { is frequency of vortex shedding }) \\
& \vec{U} \quad=\text { Air velocity vector }\left(\mathrm{ms}^{-1}\right) \\
& v_{\infty} \quad=\text { Freestream kinematic viscosity }\left(\mathrm{m}^{2} \mathrm{~s}^{-1}\right) \\
& \tilde{v} \propto S d^{2} \quad=\text { Eddy viscosity }\left(\mathrm{m}^{2} \mathrm{~s}^{-1}\right) \\
& \alpha \quad=\text { Angle of attack }\left({ }^{\circ}\right) \\
& \beta \quad=\text { Pitching angle }\left(^{\circ}\right) \\
& \gamma \quad=\text { Forewing stroke angle }\left(^{\circ}\right) \\
& \delta \quad=\text { Hindwing stroke angle }\left(^{\circ}\right) \\
& \rho=\text { Air density }\left(\mathrm{kgm}^{-3}\right) \\
& \tau=\text { Rate of strain tensor } \\
& \phi \quad=\text { Total flapping angle }\left({ }^{\circ}\right) \\
& \psi=\text { Tandem wing phase angle }\left({ }^{\circ}\right) \\
& \omega=\quad \text { Magnitude of vorticity (rps) } \\
& \Omega \quad=\text { Vorticity tensor }
\end{aligned}
$$

\section{Introduction}

Since the beginning of time, man has been intrigued by the phenomenon of natural flight. Earliest recorded evidence of engineering designs related to a natural flier were the hand drawings of Leonardo da Vinci's ornithopter in 1485. Later, Rayleigh's [1] report on soaring flight of birds, followed by Walker's [2] quantitative analysis of avian flapping flight were the most satisfactory published research on this topic. Ellington on the other hand, elaborately explained 
the insect-flight aerodynamics in a series of publications [3-5]. Generally, lift in flight is determined by wings, that deliver the high aerodynamic efficiency particularly in tandem configuration seen on locusts and dragonflies [6]-9]. There has been an extensive research focus on tandem wings using various numerical, theoretical, and experimental methods to better understand the underlying physical features facilitating their superior flight performance. Among others, Koehler et al. [10] demonstrated an experimental analysis of a dragonfly wing deformation by three-dimensional reconstruction of the insect's wings. Similarly, [11-14] digitized insect wings to investigate the effects of corrugation on the aerodynamic performance.

Although they all agree that these effects are minimal, Murphy \& Hu [15] provide further bolstering evidence on the superiority of insect wings as compared to the conventionally smooth-surfaced airfoils by measuring the flow field and vortex formation using low-speed windtunnel and digital particle image velocimetry system. These advantages include; independence of flight performance with regards to higher Reynolds number, and achievement of high maneuverability using morphing geometry [16-19]. Furthermore, Levy \& Seifert [20] demonstrate a detailed study on the influence of geometrical features of a simplified corrugated dragonfly wing at low Reynolds number $(\operatorname{Re}<8000)$.

However, dragonflies are not the only tandem winged insects with concealed aerodynamic characteristics, locusts are also mysterious enough to inspire researchers' from different domains such as computer scientists working on collision avoidance systems and swarming behavior [21-23], and aerodynamicists trying to achieve endured gliding capabilities and enhance long-range flights [24]. Since mid-20c, researchers have been compelled by these traits indeed. One of the most complete reviews on locust's steady flight aerodynamics is presented by Weis-Fogh [25] in 1956. He determined the averaged flapping angle amplitudes as $67^{\circ}$ and $109^{\circ}$ in forewings and hindwings, respectively, and concluded that the former controls flight in locusts. Although validations were not provided, later, Jensen [26] measured the stroke-averaged lift and drag generated by locusts tethered to a force balance in a wind tunnel, simultaneously recording the wing-tip motion using stroboscopic slow-motion filming. Ultimately, he confirmed that the magnitude of the aerodynamic forces are of the same order of inertial forces in both vertical and horizontal directions. Few decades later, with the development of high-speed filming, Cloupeau et al. measured the locust's wing deformation and instantaneous lift [27]. With the development of modern day technology, advanced experiments on locust wing aerodynamics unveiled much precise understanding of damping and instantaneous forces acting on locust wings [28, 29].

Despite the tremendous contributions of these researchers on the aerodynamics and kinematics of locust flight, we are yet to unveil the great potentials of this insect in conserving energy on long-range flights facilitating their distant travels [14, 24, 30]. This phenomenon is of great significance especially for natural fliers with a limited supply of food. One notable factor directly contributing to this extreme energy conservation regimen is the reduction in flapping and hence, gliding. Gliding is most famously exhibited by locusts that cross the Red Sea strait in swarms regularly. They reportedly crossed the Atlantic Ocean in ten days during the 1987-1989 plague [24].

Although continuous attempts are made to better understand the locust gliding by elucidating its aerodynamic 
footprint [14,31-33]. However, the complex deciphering of physiology and behavior of biological beings pose massive limitations in achieving satisfactory biomimetic results. Therefore, in this paper we propose an alternative by optimizing the readily digitized geometries of a locust wing cross-section profiles (airfoils) to overcome the intricacies involved in the precise digital reconstruction of invertebrates. Although there have been other alternative solutions such as energy extraction of plunging and pitching tandem wings [17], or the fusion of wing morphology and kinematics to enhance aerodynamic performance [34, 35]. Yet, there are no systematic approaches capable of yielding the desirable insect-level gliding ratio. This study aims to fulfill this gap by parameterizing the geometry of digitized locust airfoils into a precise mathematical form. To achieve this, there is a spectrum of available schemes reviewed in [36]. Commonly implemented parameterization are Hicks-Henne shape functions, Bezier curves, and B-splines. However, these are conventional methods with limited flexibility that may not satisfy the radical nature of the corrugated insect airfoils. Therefore, a more physically-intuitive scheme called the PARSEC parameterization [37, 38] is selected to serve our objective. Although certain level of adaptation is required, it is possible to transform our airfoils into a linear combination of 11 major parameters expressing its geometry as unknown base functions such as abscissa, angle of incidence, thickness, edge radius, etc.

Next, a similarly flexible shape optimization methodology is required to complement the afore mentioned parameterization scheme in realizing the ultimate goal of simultaneously and non-cooperatively optimizing multiple functions as usually demanded in enhancement of aerodynamic performance where lift and drag are directly proportional and heavily correlated. Among other popular techniques, [39] proposed the Particle Swarm optimization (PSO) that is based on the swarming of natural fliers. The method being inspired by the evolutionary programming techniques, initializes its population through random dispersion of particles with each particle retaining a personal record of its position, hence acquiring fitness from an optimization perspective. Sequential Quadratic Programming (SQP) on the other hand, is an iterative non-linear optimization technique, generally applicable to problems with twice continuously differentiable constraints and objective function [40]. However, standard Pareto Genetic Algorithms (GAs) introduced by Goldberg in 1989 [41], are usually implemented for multiple objective optimization problems similar to the one in this research. This method is further developed by Srinivas [42] and widely implemented in a range of applications. Nevertheless, use of mating or sharing constraints limits the Pareto ranking's cooperative nature. This is resolved in Nash's GA [43] that originates from Game Theory and Economics. It proposes non-cooperative multi-objective optimizations particularly satisfying our application where distinct design variables are involved.

To summarize, this study performs a 2D computational fluid dynamics analysis on the locust wing profiles (airfoils) to illustrate their basic aerodynamic performance (lift-drag ratio). These airfoils are later optimized using a novel combination of PARSEC-Nash-GA methodology described in detail. A comparative study considering the peer-models from the published literature validates the proposed optimized airfoils which are then integrated to form a 3D wing using digitized planform contours of the locust wing. Ultimately, the original as well as the optimized wings are subjected to a 
comprehensive 3D CFD analysis on a powerful cloud computing solution validating their aerodynamic performance numerically. This serves as a prerequisite for finalizing the digital wing model for further fabrication and prototyping introduced in our future study.

\section{Wing Model}

This study focuses on the gliding-expert insect, a desert locust (Schistocerca gregaria) shown in Figure 1.a). Six active and healthy (undamaged wings) farm bred adult female specimen were procured from an insect farm near Cangzhou, China. Taylor's method was followed in cementing the wing roots of the locusts in gliding posture using high viscosity Cyanoacrylate [44]. Fixed wings were later sectioned along chord at 20\%, 40\%, 60\%, and 80\% spanwise, to be primed for geometrical digitization. Thickness of the fore- and hindwings were found to be variable due to venations ranging from 2 to $3 \mu \mathrm{m}$. The pseudo-microscopic scanning of the cross-section profiles were performed by carefully placing the sectioned wings in a lightbox fixed with a mirrorless Sony Alpha A6000 SLR camera armed with an AmScope 45x stereo microscope.

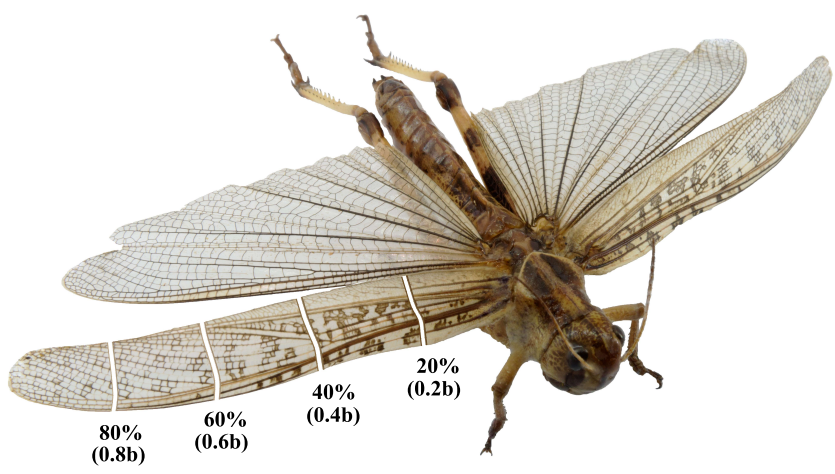

(a)

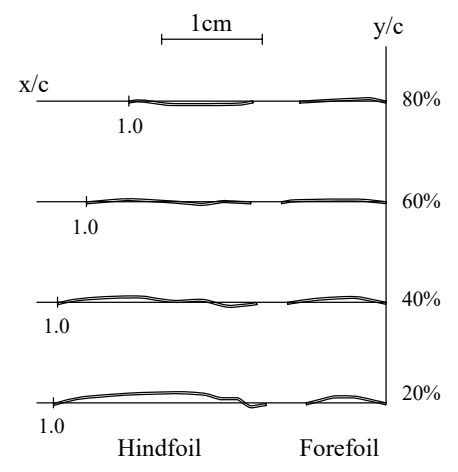

(b)

Fig. 1 Graphical representation of the locust wing sectioned along chord at $20 \%, 40 \%, 60 \%$, and $80 \%$ spanwise from wingroot. (a) illustration of the sectioned wings, and (b) 2D CAD fore- and hindfoil geometries representing locust airfoils.

Our digitized profiles were validated against the locust-airfoil geometries recorded by Walker et al. [31], in a smoke flow visualization experiment. As shown in Figure 1 (b), the CAD-based geometries of the wing profiles extracted from the recorded image files closely correlate with the observations from literature [31]. These geometries are primed for 2D CFD simulations without alterations and modification at this stage. It must be noted that this study mainly focuses on the development of an optimal gliding wing prototype, hence in accordance with the related literature [13-15], the effects of insect body and thickness variations due to wing venations are assumed negligible.

Additionally, the wings in all three directions (front, top, and side) were recorded prior to sectioning in order to facilitate a pseudo-microscopic 3D reconstruction of the wings involving every crucial detail such as corrugations and 
fore-hindwing transverse \& longitudinal spacing. Ultimately, to form the 3D wing structure in SolidWorks, a thin solid boss section was swept through the wing cross-section profiles (chord) at 20\%, 40\%, 60\%, and 80\% spanwise represented as $0.2 b, 0.4 b, 0.6 b$, and $0.8 b$, where $b$ is the wingspan along the contour (guide curve) defined by the locust wing planform shown in Figure 1 (a).

\section{A. Numerical Methods}

The 2- and 3D numerical studies are developed here as a test bed to endorse our proposed wing optimization methodology and its effectiveness that shall further facilitate prototyping of bio-inspired artificial wings for flying micro robot applications. Here, the airfoil and wing thicknesses are standardized to $0.02 \%$ mean chord length acting as rigid bodies similar to the published models [14, 45]. In contrast to the literature [35, 46] however, our access to a powerful computing engine with 96x 3.4GHz Intel Kabylake cores processing on 360GB of RAM sponsored by the Google Cloud Platform, facilitated an accurate 3D CFD analysis on a detailed and high-resolution corrugated digital locust wing.

For a successful fluid mechanics simulation, configurations such as compressibility and nature of flow, flight regime deciding Reynolds number $(R e)$, domain size, and boundary conditions must be clearly defined. Most insects fly at lower subsonic flight regime, to which locust is no exception. Hence, nature of the fluid flow around its wings remains turbulent and incompressible solved with a pressure-based solver. A volumetric flow field at low Reynolds number is mostly governed by the 3D Navier-Stokes equations [47], with the Reynolds number itself calculated as, $R e=U l / v_{\infty}$, where $l$ is the characteristics length, $v_{\infty}$ is the freestream kinematic viscosity, and $\vec{U}=(U V W)$ is the velocity vector of the fluid. Furthermore, the lift force that is perpendicular to the direction of fluid flow (x-axis in this case) is calculated as $C_{L}=\frac{2 L}{\rho U^{2} S}$, drag is $C_{D}=\frac{2 D}{\rho U^{2} S}$, and the pressure coefficient given by $C_{P}=\frac{P-P_{\infty}}{0.5 \rho U^{2}}$, where, $\rho$ is the fluid density, $L$ is the lift, $D$ is the drag on the airfoil, $S$ is reference area (for airfoil; $S=c(1)=c$ ), and $P_{\infty}$ is the freestream pressure.

Gliding flight is ideally associated with a laminar flow, however, natural fliers are exceptions due to their wing corrugations and intermittent flapping that creates sustained turbulence [48] defined here separately for 2- and 3D wing as, (a) the Spalart-Allmaras model [49] solving Reynolds-averaged Navier-Stokes (RANS) equations for the 2D analysis, and (b) the Spalart-Allmaras Delayed Detached Eddy Simulation (SA-DDES) model for the 3D wing. The Spalart-Allmaras turbulence model is given as,

$$
\begin{array}{r}
\frac{\partial \tilde{v}}{\partial t}+u_{j} \frac{\partial \tilde{v}}{\partial x_{j}}=c_{b 1}\left(1-f_{t 2}\right) \tilde{\omega} \tilde{v}-\left[c_{w 1} f_{w}-\frac{c_{b 1}}{\kappa^{2}} f_{t 2}\right] \\
\left(\frac{\tilde{v}}{d}\right)^{2}+\frac{1}{\sigma}\left[\frac{\partial}{\partial x_{j}}\left((v+\tilde{v}) \frac{\partial \tilde{v}}{\partial x_{j}}\right)+c_{b 2} \frac{\partial \tilde{v}}{\partial x_{i}} \frac{\partial \tilde{v}}{\partial x_{i}}\right]
\end{array}
$$

where $v$ is the molecular kinematic viscosity and $\tilde{v}$ obeys the transport equation, $\omega$ is the magnitude of vorticity, $d$ is the distance to the closest wall, and $c_{b 1}, c_{b 2}, \kappa$, and $\sigma$ are constants. 
The relation between the transport variables, $\tilde{v}$ and $\tilde{\omega}$ is given as;

$$
\tilde{\omega}=\omega+\frac{\tilde{v}}{\kappa^{2} d^{2}} f_{v 2}, \quad f_{v 2}=1-\frac{\chi}{1+\chi f_{v 1}}
$$

It can be noted that wall boundary condition is $\tilde{v}=0$ for freestream flows. Detailed definition of all the terms, derivations, and the magnitude of the constants are described in the literature [49].

However, for the 3D wing turbulence, Spalart et al. modified the above model by Detached Eddy Simulation (DES) formulation where the wall distance $d$ was replaced by the modified distance function given as,

$$
l_{D E S}=\min \left(d, C_{D E S} \Delta\right), \Delta=\max (\Delta x, \Delta y, \Delta z)
$$

where $\Delta$ is the filter width in large eddy simulations and $C_{D E S}=0.65$ is an empirical constant. The S-A model contains both a destruction as well as a production term that when balanced, adjusts the eddy viscosity $\tilde{v}$ to scale with the local deformation rate $S$ and $d$ deriving the relation, $\tilde{v} \propto S d^{2}$. The fundamental goal behind Delayed Detached Eddy Simulation (DDES) is to calculate a scale in DES $\left(l_{D E S}\right)$ that shall delay the switching of RANS to LES mode near the boundary layer.

Solver methods and schemes for spatial discretization and pressure-velocity coupling are selected as second order upwind and pressure implicit with splitting of operator (PISO), respectively. To bolster the simulations, further temporal accuracy for the solutions are obtained using two-step implicit expression law involving an extra time-step on all iterations. Initial conditions are selected to be uniform according to the inlet fluid flow with numerical accuracy set to triple-precision.

\section{B. Boundary Conditions and Discretization}

The initial step for an intuitive simulation study is to create a computational domain and discretize it for the solver engine. Here, we propose a CO- and CV-type domains that are sufficiently large to enclose the locust airfoils and wings, respectively. $\mathrm{CO}$ and $\mathrm{CV}$ are the notations usually used to describe the shape of computational domains in $\mathrm{CFD}$. The 2D domain is described in [50], however, the volume mesh for our 3D wing is seen in Figure 2 measuring 15c (wing chord length) in both vertical and horizontal directions. The main objective of these strict dimensioning is to reduce the domain wall influence on the flow field around the test subject and the turbulence models defined. The inlet condition is set as the dome shaped face opposite to the wing leading-edge (LE), and the outlet is set as the flat face opposite to the wing's trailing-edge (TE). The wing itself is considered as the subject wall in a gliding posture defined in the later sections. The Reynolds number $(R e)$ for all the CFD simulations in this study is determined using the relation $\left(R e=U l / v_{\infty}\right)$, where the freestream kinematic viscosity $\left(v_{\infty}\right)$ is calculated by dividing the dynamic viscosity of the 


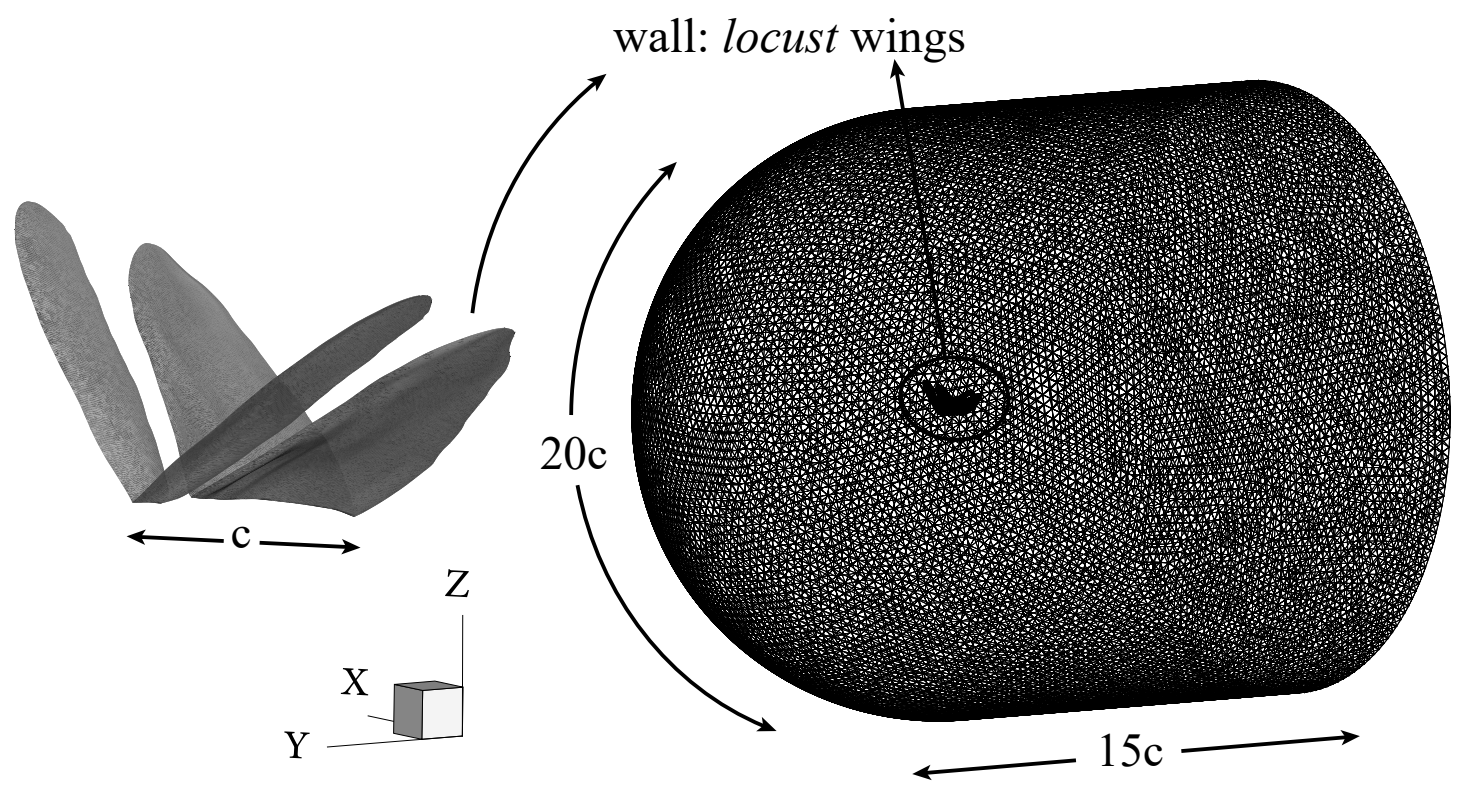

Fig. 2 Schematic representation of the 3D meshed locust wing within a hybrid CV-type computational domain setup with the related boundary conditions.

fluid with its density. Also, $R e$ remains within the range of 10,000 to abide by the locust's relatively low flight velocity also defined in the literature [14, 20].

Furthermore, well-calibrated volume grids are generated for the computational domain using ANSYS meshing. This is shown in Figure 2 where the CV-type domain encloses the digitized locust wing with an approximately 108 million elements mainly concentrated near the boundary layer and critical regions such as the corrugations to obtain a satisfactory orthogonal quality. This was achieved with the help of our hybrid design CV-type domain that featured a V-shaped geometry enclosing the wings with a small separation that acted as the body-of-influence to obtain local element size smaller than $0.3 \mathrm{~mm}$. Using this domain at $\alpha=0^{\circ}$ and $R e=7000$, the coefficient of drag and lift converged at $1.01 \times 10^{-4}$ and $6.82 \times 10^{-4}$, respectively. It must be noted that the angle of attack $(\alpha)$ throughout this study is the angle between the relative fluid flow and the chord (c) of the fore- and hindwing synchronized (i.e. angle of attack for fore- and hindwing always remains same unless stated otherwise). Various sizes of grids were explored to determine the most balanced trade-off strategy between the solution accuracy and mesh resolution. It was found that the grids with a size below $0.3 \mathrm{~mm}$ had an insignificant (less than $2.5 \%$ ) effect on the converged aerodynamic forces.

\section{Optimization Methodology}

This study considers the digitized locust wing cross-section profile as a conventional fixed-wing airplane airfoil with no special treatment contrary to the literature [14, 17, 35]. Therefore, a combination of pragmatic aeronautical-specific optimization methodologies is proposed to enhance its aerodynamic efficiency. This combination is a hybridization of Nash's strategy with the bio-inspired Genetic algorithms (GA) accompanied by a PARSEC parameterization scheme 
validated against a published procedure [51] in terms of solution accuracy and computational efficiency. In particular, the bio-inspired nature and the versatility of this scheme well accommodates our unconventionally shaped (corrugated) airfoils. Ultimately, the optimized airfoils are integrated to form a whole-optimized bio-inspired digital wing.

\section{A. Nash Genetic Algorithm}

In the context of Game Theory, Nash introduced a non-cooperative multi-objective optimization methodology in 1951 [43]. He suggested the use of players as distinct objectives for an optimization problem (the game), where no player can have an individual gain by changing their strategy unilaterally [52]. In a two player game, Nash's equilibrium $N$ which is the 'optimized state' is achieved when no further enhancement of criterion is possible by either of the players, mathematically represented as,

$$
\left\{\begin{array}{l}
\text { Determine }\left(\bar{p}_{1}, \bar{p}_{2}\right) \in P_{1} \times P_{2} \quad \text { such that } \\
f_{1}\left(\bar{p}_{1}, \bar{p}_{2}\right)=\min _{p_{1} \in P_{1}} f_{1}\left(p_{1}, \bar{p}_{2}\right) \quad \text { and } \\
f_{2}\left(\bar{p}_{1}, \bar{p}_{2}\right)=\min _{p_{2} \in P_{2}} f_{2}\left(\bar{p}_{1}, p_{2}\right)
\end{array}\right.
$$

where $f_{1}$ and $f_{2}$ are the objective functions (players), and $\left(p_{1}, p_{2}\right) \in P_{1} \times P_{2}$ are their strategies defined in a local domain. Particularly, $p_{1}$ and $p_{2}$ are each a group of variables defined by $p_{1}=\left[\gamma_{1} \ldots, \gamma_{n}\right], p_{2}=\left[\eta_{1} \ldots, \eta_{m}\right]$. The dimension of this group $(n, m)$ is defined in the parameterization stage later.

On the other hand, the second optimization method introduced in this study is the bio-inspired Genetic algorithms, that is based on the concept of genetics and natural selection. Adaptive heuristic search approach is implemented in this method where a generation representing an iteration of an optimization problem can evolve only if its members (or players) have satisfied certain conditions which is either maximization or minimization of an objective or a cost function, respectively [53]. A general composition of a GA is given as;

- a genotype that is a string of bits representing a population of individuals with an assigned finite size,

- a fitness function that is a measure of an individual's fertility, and adaptability to the environment,

- a set of random genetic operators such as mutation, selection, and crossover.

Several salient merits of the GAs are; (a) compatibility with experimental or numerical data, (b) nature-inspired parallel processing, (c) use of continuous or discrete variables, and (d) ability to handle large number of variables with complex cost functions (surfaces in our application).

Having justified the suitability of Nash strategy and Genetic algorithms for our application, we hybridize the two schemes similar to the work presented by D'Amato et al. [54, 55], where a two-player game strategy based on Nash equilibrium that defines the two players' set of variables as $X, Y$ whose real valued functions $f_{1}, f_{2}$ represent either objective or cost function of the players. According to Fudenberg's [56] adjustment process, players decide their 


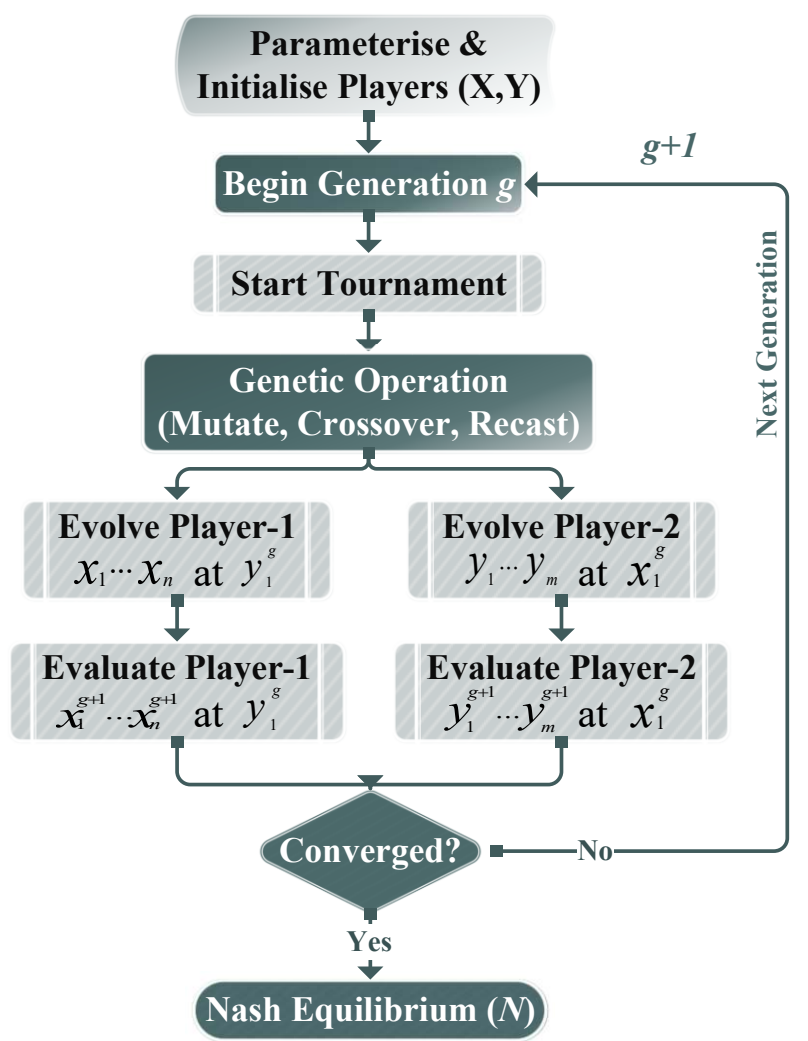

Fig. 3 Illustration of the process flow for the hybridized Nash-GA optimization algorithm.

intended outcome sequentially to make sure it is an improvement compared to their opponent's latest outcome until the Nash equilibrium state is reached which is also the converged solution. Following the notion from Genetics, this solution is denoted as a chromosome which is a representation of a string, $s=y, x$ where $y$ and $x$ are both a subset of variables grouped as players $\left(p_{2}, p_{1}\right)$ within the metric space $(Y, X)$, respectively.

Hence, we can conclude that the hybridized method can reach the state of equilibrium, $N$, when the string, $s$ is optimized by player- 2 modifying $y$ with respect to $f_{2}$ while $x$ is fixed, and player- 1 optimizes the chromosome with respect to $f_{1}$ by fixing $y$ and altering $x$ [43]. Applying this procedure here as shown in Figure 3 , player-1 optimizes $x^{g}$ using $y^{g-1}$ in order to evaluate the chromosome $\left(s=x^{g}, y^{g-1}\right)$, while simultaneously, player-2 is optimizing $y^{g}$ using $x^{g-1}$ to evaluate its own chromosome $\left(s=x^{g-1}, y^{g}\right)$.

Each player is assigned with a random population of individuals responsible for the execution of the optimization process for that player. The matching outputs of individuals from each player are classified based on their fitness 
represented by 0 for draw, and -1 or 1 for lose or win, respectively.

$$
\begin{cases}\text { if } \quad f_{1}\left(x_{i}^{g}, y^{g-1}\right)>f_{1}\left(x^{g-1}, y_{i}^{g}\right), & \text { fitness }=1 \\ \text { if } \quad f_{1}\left(x_{i}^{g}, y^{g-1}\right)<f_{1}\left(x^{g-1}, y_{i}^{g}\right), & \text { fitness }=-1 \\ \text { if } \quad f_{1}\left(x_{i}^{g}, y^{g-1}\right)=f_{1}\left(x^{g-1}, y_{i}^{g}\right), & \text { fitness }=0\end{cases}
$$

And same holds for player- 2 where only $f_{1}$ is replaced by $f_{2}$,

$$
\begin{cases}\text { if } \quad f_{2}\left(x_{i}^{g}, y^{g-1}\right)>f_{2}\left(x^{g-1}, y_{i}^{g}\right), & \text { fitness }_{2}=1 \\ \text { if } \quad f_{2}\left(x_{i}^{g}, y^{g-1}\right)<f_{2}\left(x^{g-1}, y_{i}^{g}\right), & \text { fitness }_{2}=-1 \\ \text { if } \quad f_{2}\left(x_{i}^{g}, y^{g-1}\right)=f_{2}\left(x^{g-1}, y_{i}^{g}\right), & \text { fitness }_{2}=0\end{cases}
$$

Such sorting criterion is precise and clearly groups individuals from population 2 on $f_{2}$ and vice-versa, for an equivalent value of fitness. Each player's population undergoes common GA techniques such as mutations and crossover once the parent chromosomes form their mating pool. A second sorting procedure is needed after this evolution process, in order to classify the new population made of parents and offsprings together. The number of parts in a chromosome is decided by the number of participating players. In this case, part one is generated by player- 2 at generation $g+1$ using the player-1's best value $x^{g}$ and the second part is generated by players-1 at generation $g+1$ using the player-2's best value $y^{g}$. This is repeated until the solution has converged or the terminal period limit reached, which is also called as the Nash equilibrium $(N)$ state.

\section{B. Nash GA-PARSEC Fusion}

To proceed with the application of the above hybrid optimization technique, we must first transform the digitized airfoil from its physical form into a mathematical one comprising of a number of variables defining its geometrical shape. For this, we adapt Sobieczky's [38] airfoil-specific parameterization technique which is fairly flexible and reliable. It is based on the concept of representing an airfoil's geometry as a linear combination of base functions that are unknown with eleven major parameters associated with the well-identified shape attributes of an airfoil as shown in Figure 4 and described in Table 1

These parameters must be selected such that, upon solving them as a linear system, the final airfoil shape must be determined. Mathematically, the formulation of PARSEC can be expressed as,

$$
v_{t}=\sum_{i=1}^{n=6} j_{t}^{i} \cdot u^{i-0.5}, \quad v_{b}=\sum_{i=1}^{n=6} j_{b}^{i} \cdot u^{i-0.5}
$$




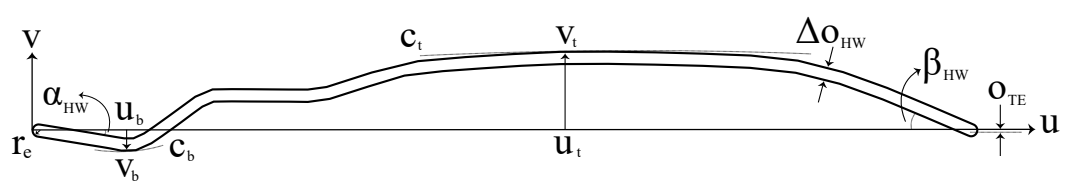

(a)

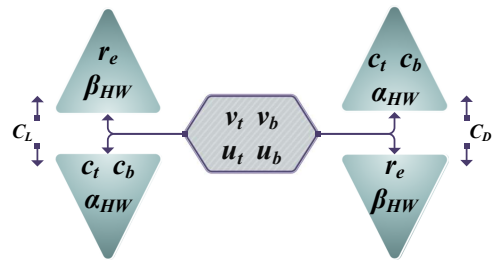

(b)

Fig. 4 Illustration of the PARSEC parameterization and Nash-GA hybridization; (a) PARSEC parameterization adapted for a locust-inspired hindfoil (b) depiction of design variables and their level of dependency on each player $\left(C_{L}\right.$ or $\left.C_{D}\right)$. Adapted with permission from [50].

Table 1 List and description of the PARSEC parameters corresponding to the bio-inspired airfoil's defining attributes

\begin{tabular}{lcc}
\hline PARSEC & GEOMETRY & DESCRIPTION \\
\hline$a_{1}$ & $r_{e}$ & edge radius \\
$a_{2}$ & $u_{t}$ & top crest horizontal position \\
$a_{3}$ & $v_{t}$ & top crest vertical position \\
$a_{4}$ & $c_{t}$ & top crest curvature \\
$a_{5}$ & $u_{b}$ & bottom crest horizontal position \\
$a_{6}$ & $v_{b}$ & bottom crest vertical position \\
$a_{7}$ & $c_{b}$ & bottom crest curvature \\
$a_{8}$ & $o_{T E}$ & trailing edge vertical offset \\
$a_{9}$ & $\Delta o_{H W}$ & profile thickness \\
$a_{10}$ & $\alpha_{H W}$ & leading-edge angle of incidence \\
$a_{11}$ & $\beta_{H W}$ & trailing edge angle of incidence \\
\hline
\end{tabular}

where $v_{t}, v_{b}$ are the vertical coordinate of the upper and lower surface, respectively, and $u$ is the chord-wise coordinate normalized in $[0,1]$. The coefficients $j_{t}, j_{b}$ are determined using the 11 defined parameters as follows:

$$
l_{t} \times j_{t}=k_{t}, \quad l_{b} \times j_{b}=k_{b}
$$

where the above coefficient matrices $\left(l_{t}, l_{b}\right)$ and $\left(k_{t}, k_{b}\right)$ are defined by separate matrices given as;

$$
l_{t}=\left|\begin{array}{cccccc}
1 & 1 & 1 & 1 & 1 & 1 \\
a_{2}^{\frac{1}{2}} & a_{2}^{\frac{3}{2}} & a_{2}^{\frac{5}{2}} & a_{2}^{\frac{7}{2}} & a_{2}^{\frac{9}{2}} & a_{2}^{\frac{11}{2}} \\
\frac{1}{2} & \frac{3}{2} & \frac{5}{2} & \frac{7}{2} & \frac{9}{2} & \frac{11}{2} \\
\frac{1}{2} a_{2}^{-\frac{1}{2}} & \frac{3}{2} a_{2}^{\frac{1}{2}} & \frac{5}{2} a_{2}^{\frac{3}{2}} & \frac{7}{2} a_{2}^{\frac{5}{2}} & \frac{9}{2} a_{2}^{\frac{7}{2}} & \frac{11}{2} a_{2}^{\frac{9}{2}} \\
-\frac{1}{4} a_{2}^{-\frac{3}{2}} & \frac{3}{4} a_{2}^{-\frac{1}{2}} & \frac{15}{4} a_{2}^{\frac{1}{2}} & \frac{15}{4} a_{2}^{\frac{1}{2}} & \frac{63}{4} a_{2}^{\frac{5}{2}} & \frac{99}{4} a_{2}^{\frac{7}{2}} \\
1 & 0 & 0 & 0 & 0 & 0
\end{array}\right|
$$




$$
k_{t}=\left|\begin{array}{c}
a_{8}+\frac{a_{9}}{2} \\
a_{3} \\
\tan \left(a_{10}-\frac{a_{11}}{2}\right) \\
0 \\
\frac{a_{4}}{\sqrt{2 a_{1}}}
\end{array}\right|, \quad k_{b}=\left|\begin{array}{c}
a_{8}-\frac{a_{9}}{2} \\
a_{6} \\
\tan \left(a_{10}+\frac{a_{11}}{2}\right) \\
0 \\
\frac{a_{4}}{\sqrt{2 a_{1}}}
\end{array}\right|
$$

where coefficient matrices $k_{t}$ and $k_{b}$ differ by $a_{3} / a_{6}$ and an addition/subtraction operation whereas, the matrices $l_{t}$ and $l_{b}$ simply vary by a subscript ' 5 ' in eq. 9 , i.e. all the $a_{2}$ s become $a_{5}$ for the $l_{b}$ matrix.

The implementation of this non-cooperative multiple objective optimization process facilitates a simultaneous maximization/minimization of two separate functions called players. Here, the two players represent the coefficients of drag and lift that are assigned with different physical Design Variables (DVs) as shown in Figure 4 b). Each player $\left(C_{L} \&\right.$ $C_{D}$ ) has certain level of dependency on different DVs that is schematically illustrated in Figure 4 b), and interpreted as; player-2 $\left(C_{D}\right)$ majorly dependent on leading-edge (LE) angle of incidence $\left(\alpha_{H W}\right)$, LE radius $\left(r_{e}\right)$, and profile thickness $\left(\Delta o_{H W}\right)$, while player-1 $\left(C_{L}\right)$ mainly depends on trailing-edge (TE) angle of incidence $\left(\beta_{H W}\right)$, TE vertical offset $\left(o_{T E}\right)$, lower crest curvature $\left(c_{b}\right)$, and the top crest curvature $\left(c_{t}\right)$. Several DVs on the other hand, are equally influential on both players. These are, the top and bottom crest horizontal positions $\left(u_{t}, u_{b}\right)$, and the vertical positions of top and bottom crests $\left(v_{t}, v_{b}\right)$. However, two parameters being the profile thickness $\left(\Delta o_{H W}\right)$ and the TE vertical offset $\left(o_{T E}\right)$ remain unaltered to reduce the computational cost of the algorithm.

In order to evaluate the Nash equilibrium $(N)$ with different strategies, we must consider all combinations possible for a PARSEC parameterization. However, to limit the overextension of this paper, assignment of common Design Variables (cDVs) are prioritized to deduce sixteen combinations possible, calculated as,

$$
C_{n=4, p=2}=\sum_{i \in n_{0}} \frac{n !}{i !(n-1) !}=16
$$

where $p$ is the number of players, $n$ is the number of common DVs $\left(v_{b}, v_{t}, u_{t}\right.$, and $\left.u_{b}\right)$, and $n_{0}=[0, \ldots, n]$. Table 2 lists all sixteen strategies (combinations) composed of different common DV assignments. It must be noted that each player is assigned only with its corresponding highly influential DV. Additionally, we reduce the evaluation process further by classifying the strategies into 5 groups of; 4L-0D, 3L-1D, 2L-2D, 1L-3D, and $0 \mathrm{~L}-4 \mathrm{D}$, where ' $\mathrm{L}$ ' and ' $\mathrm{D}$ ' represent players 1 and 2 (i.e. lift and drag), and the numerical prefixes indicate the number of common DVs assigned to their corresponding player. 
Table 2 A list of combinations for input Nash-GA based variables

\begin{tabular}{ccc}
\hline $\begin{array}{c}\text { Combination } \\
\text { (strategy) }\end{array}$ & $\begin{array}{c}\text { Player-1 }\left(C_{L}\right) \\
c_{t}, c_{b}, o_{T E}, \beta_{H W}\end{array}$ & $\begin{array}{c}\text { Player-2 }\left(C_{D}\right) \\
r_{e}, \Delta o_{H W}, \alpha_{H W}\end{array}$ \\
\hline 1 & $v_{t}, u_{b}, v_{b}$ & $u_{t}$ \\
2 & $v_{t}, u_{b}$ & $u_{t}, v_{b}$ \\
3 & $v_{t}, v_{b}$ & $u_{t}, u_{b}$ \\
4 & $v_{t}$ & $u_{t}, u_{b}, v_{b}$ \\
5 & $u_{b}, v_{b}$ & $u_{t}, v_{t}$ \\
6 & $u_{b}$ & $u_{t}, v_{t}, v_{b}$ \\
7 & $v_{b}$ & $u_{t}, v_{t}, u_{b}$ \\
8 & - & $u_{t}, v_{t}, u_{b}, v_{b}$ \\
9 & $u_{t}, v_{t}, u_{b}, v_{b}$ & - \\
10 & $u_{t}, v_{t}, u_{b}$ & $v_{b}$ \\
11 & $u_{t}, v_{t}, v_{b}$ & $u_{b}$ \\
12 & $u_{t}, v_{t}$ & $u_{b}, v_{b}$ \\
13 & $u_{t}, u_{b}, v_{b}$ & $v_{t}$ \\
14 & $u_{t}, u_{b}$ & $v_{t}, v_{b}$ \\
15 & $u_{t}, v_{b}$ & $v_{t}, u_{b}$ \\
16 & $u_{t}$ & $v_{t}, u_{b}, v_{b}$ \\
\hline
\end{tabular}

\section{Results}

The Nash-GA based optimization method proposed in this study is used to improve all the digitally-reconstructed airfoils inspired by the real locust fore- and hindwing. The optimized as well as the unoptimized airfoils are integrated to form two 3D wings for further aerodynamic analysis. Therefore, to extend the scope of this study, performance of the wings along with one of the airfoils $(0.2 \mathrm{~b})$ is discussed in detail.

Contrary to the literature [14], here the optimization and fluid dynamic studies are performed on the airfoils in tandem configuration to take the aerodynamic interaction of fore- and hindfoils into consideration. The original PARSEC parameters for all the airfoils are collectively reduced by $25 \%$ as an attempt to optimize them uniformly without aerodynamic and geometric constraints. Also, due to radical nature of the geometries and DVs involved in this study, a one-size-fits-all automated procedure may not be implemented. Therefore, the entire optimization process is operated and monitored manually on an individual-case basis.

A series of 2- and 3D computational fluid dynamics modeling is performed in this section to explore the aerodynamic performance of the two wings and airfoil, $0.2 \mathrm{~b}$. The fluid flow is viscous, steady, and incompressible at different angles of attack flowing with a velocity, $0.6-6 \mathrm{~ms}^{-1}(R e=7000)$. Furthermore, the study involves both qualitative and quantitative analysis to perform an exhaustive evaluation against the original wings and the literature. With the help of a conventional airfoil (NREL S809) [51], the proposed optimization algorithm is validated prior to implementing it on our bio-inspired wing cross-section profiles. This validation is graphically represented in Figure 5 (a), where an evident link 


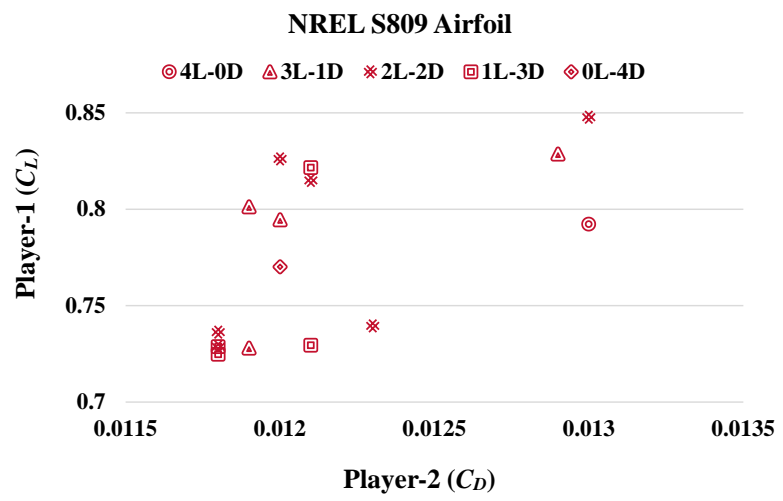

(a)

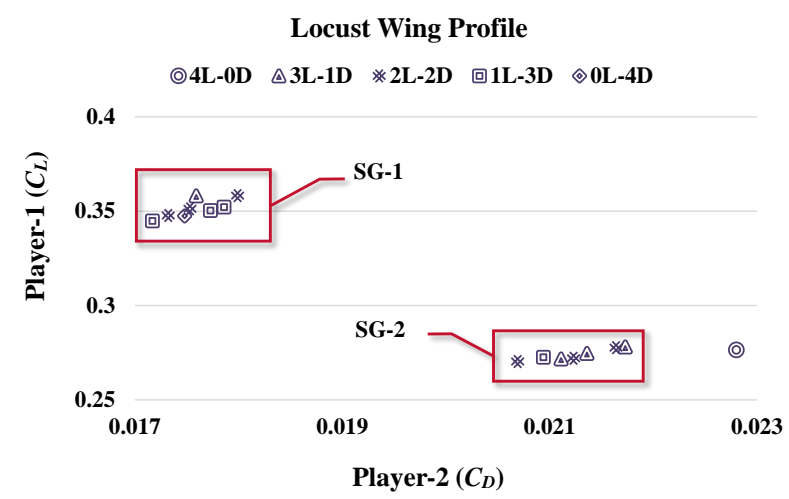

(b)

Fig. 5 Graphical representation of the Nash equilibria for a two player optimization determined as a set of points plotted for (a) a conventional NREL-S809 airfoil, and (b) a locust-inspired digitized airfoil (0.2b). Adapted with permission from $[\mathbf{5 0}]$.

is formed between the obtained points for the Nash equilibria set. In contrast to the conventional smooth-surface airfoils, the established links for bio-inspired airfoils are inversely proportional, that is each player with the maximum number of common DVs assigned, would achieve the least objective. Therefore, a set of Nash equilibria points are obtained for the locust-inspired digitized airfoils. The points are clustered in two widely spread zones called SG-2 and SG-1 with combinations delivering maximum drag/minimum lift and maximum lift/minimum drag, respectively.

This is graphically represented for the sample locust airfoil at $0.2 \mathrm{~b}$ in Figure 5 (b) facilitating a convenient establishment of a fine trade-off strategy between the two players. It is evident from the graph that the locust airfoils are much similar to a conventional smooth-surface airfoils in terms of achieving minimum drag and maximum lift when a strategy has most of its cDVs assigned to the player-2 or 1, respectively. This validates the proposed Nash-GA based bio-inspired optimization algorithm applied on the corrugated locust-inspired digitized airfoils in this study. Nevertheless, a comprehensive performance evaluation presented in the following section is necessary to explore the full potentials of this methodology in the biomimetic aircraft industry.

\section{A. Aerodynamic Forces}

Initially, we proceed with the quantitative study of the locust wing aerodynamic performance. The numerical data involved are basically the lift and drag coefficients $\left(C_{L} \& C_{D}\right)$ that are crucial aerodynamic characteristics of a wing in general. It is found that the locust-inspired airfoils prior to optimization, are already better performing with a large margin compared to the same airfoils from the published research [14]. This difference in aerodynamic performance (gliding ratio) exceeds $600 \%$ which is speculated to be due to peer model's extremely curved profiles especially in the case of forefoils (extended top crest vertical position) that may cause the steep elevation of induced drag. Contrary to the reported bio-inspired airfoil performances at low $R e$, our digitally reconstructed locust airfoils are comparable to 
their smooth-surfaced conventional counterparts from the aircraft industry. As an example, considering the highest achievable aerodynamic efficiency (gliding ratio) for the locust-inspired airfoil at $20 \%$ spanwise $(0.2 \mathrm{~b})$ plotted on the graph in Figure 6a), is 40\% smaller than NACA 0012's best performance $\left(\eta_{a}=25.69\right)$ at $\alpha=5^{\circ}$ and $R e=50,000$, and $75 \%$ greater than the best efficiency $\left(\eta_{a}=8.7451\right)$ determined for a conventional slow-flight Gottingen 501 airfoil at $R e=50,000$ and $\alpha=4^{\circ}$, with a maximum thickness of $12.8 \%$ at $30 \%$ chord and maximum camber of $6.3 \%$ at $50 \%$ chord [57]. Also, at low Re the stall margin remains within the range of $2-4^{\circ}$ for both the corrugated airfoils as well as the conventional ones. Considering the difference in $R e$ values further proves the exceptional performance of these bio-inspired airfoils for slow-flight applications.

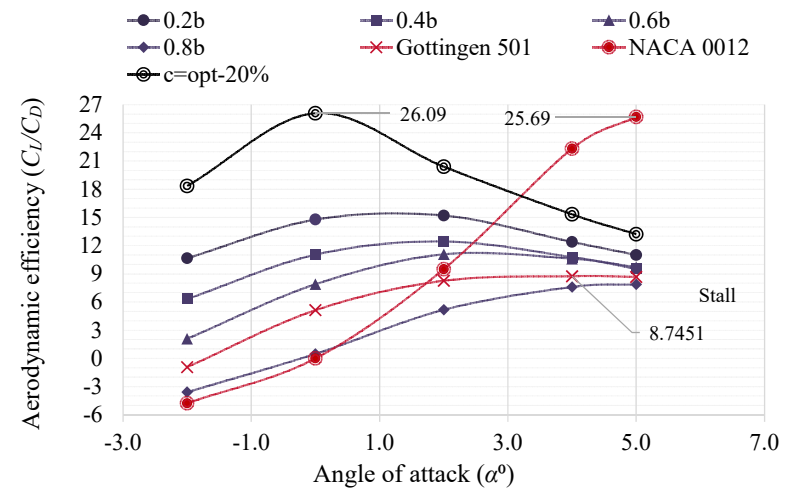

(a)

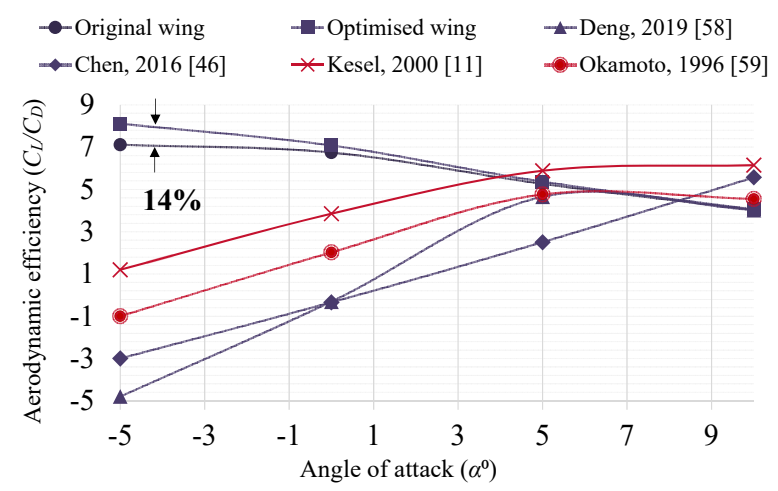

(b)

Fig. 6 Graphical plot of the gliding ratio $\left(\eta_{a}\right)$ for; (a) locust-inspired digitized airfoils at $20 \%, 40 \%, 60 \%$, and $80 \%$ spanwise, and (b) the optimized and unoptimized 3D wings at different angles of attack with $R e=7000$.

The optimized locust-inspired airfoil at $20 \%$ spanwise $(0.2 \mathrm{~b}$-opt $)$ on the other hand, delivers a $77 \%$ improved performance (gliding ratio) when compared to its unoptimized counterpart at zero degree angle of attack. This reiterates the afore mentioned claim that the improvement of a player's performance is heavily dependent on the number of cDVs assigned to it. Therefore, the airfoils with minimum drag (most cDVs for player-2) are well streamlined with a sharper tip and a slimmer profile, whereas the airfoils with maximized lift (most cDVs for player-1) possess longer suction portions with thicker profiles. This necessitates the establishment of a balanced trade-off strategy between the two players to achieve maximum lift without compromising much on the drag. Comparing the performance of all the airfoils from all the strategies, strategy-1 airfoil resulting from the 1L-3D group (SG-1 zone) best delivers such balanced design trade-off. With a lift coefficient of 0.2781 at a negligible $0.0171 \mathrm{drag}$, indicates that strategy- 1 is mainly focused on drag reduction to enhance the airfoil's aerodynamic efficiency. This is due to the limited possibility of raising lift coefficient beyond $4 \%$ by any of the strategies. Hence, the strategy- 1 with maximum decrease in drag $(25 \%)$ succeeds.

Similarly, precise trade-off strategies were established for all the airfoils, producing a bio-optimized bio-inspired locust tandem wing, whose performance is compared to the bio-inspired 3D wing performances in the literature [46, 58, 59], shown in Figure 6(b). Both the original and the optimized wings deliver higher gliding ratios at lower 


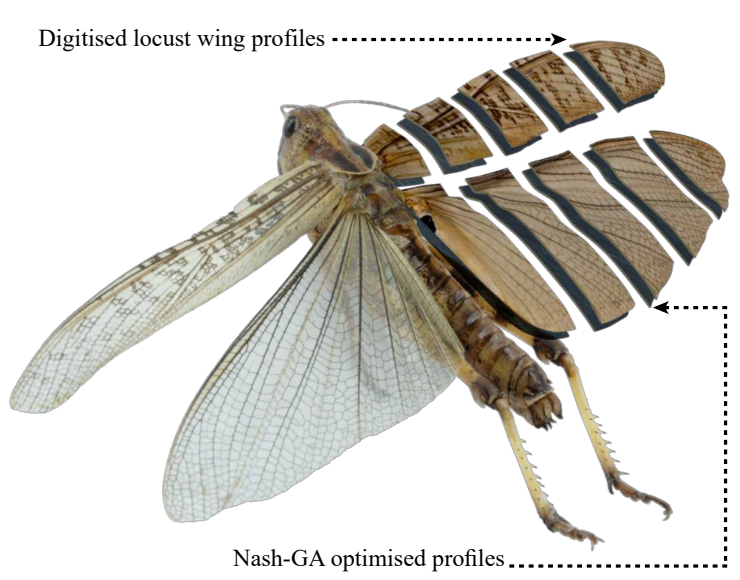

(a)

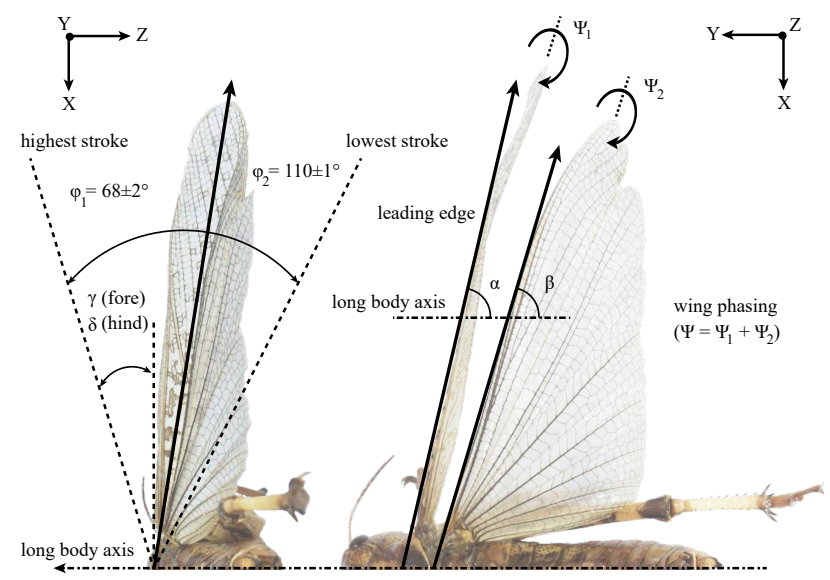

(b)

Fig. 7 Graphical representation of the 3D reconstructed locust wing. (a) original (upper) and the strategy-1 (lower) wings superimposed, (b) 3D wing orientation parameters to define locust wing gliding posture.

angles of attack by a factor of 7x. However, the Nash-GA optimized wing as a whole delivers $14 \%$ improvement compared to its unoptimized version at $\operatorname{Re}=7000$ and $\alpha=-5^{\circ}$. Nevertheless, the analysis of the 3D wings did not involve geometry scaling and the wings were positioned in the gliding posture described above. Therefore, a definitive conclusion on the performance can only be achieved in an experimental evaluation (PIV measurement) of the fabricated prototypes [60].

The digitally reconstructed locust-inspired 3D wing and its strategy-1 optimized counterpart are superimposed and illustrated in Figure 7 (a). The 25\% reduction in common DV's is visually evident in the figure where changing $v_{t}=1.184, v_{b}=0.231$, and $u_{b}=1.464$ to $v_{t}=0.888, v_{b}=0.1738$, and $u_{b}=1.098$ has resulted in further profiling (streamlined) of the external geometry while limiting the available suction regions on the Nash-GA optimized (lower) wing. And due to the proportional profiling of the optimized wing, precipitous changes in corrugations gradually decrease spanwise. On the other hand, Figure 7(b) illustrates the orientation parameters required to define gliding posture of the locust wing. Here, we follow Baker's observation of the real insect in gliding mode [61]. Where the wing orientation is set as, (a) forewing; $\psi_{1}=5^{\circ}$ in X-plane, $\gamma=-35.7^{\circ}$ in Y-plane, $\alpha=-10^{\circ}$ in Z-plane, and (b) hindwing; $\psi_{2}=9^{\circ}$ in X-plane, $\delta=-34.8^{\circ}$ in Y-plane, $\beta=-10^{\circ}$ in Z-plane. The angles are set about the wingroot in the global XYZ coordinate system.

\section{B. Pressure $\left(C_{P}\right)$ Distribution}

Pressure distribution over the airfoils and wings is mainly analyzed; to validate our digital reconstruction, optimization process, and to determine aerodynamic effects of various regions (corrugations) on the flow field. As for the validations, Bernoulli's principle is satisfied for both the optimized as well as the unoptimized airfoils shown in Figures 9 (a) and 8 (a), respectively. This is proven by a clear color distinction indicating higher (red) and lower (blue) gradients of pressure 
spread over the bottom and top surface of the airfoil surface, respectively. Airfoils forming the wings here are previously validated in [50]. As seen in Figure 10, the Bernoulli's validation holds good for the 3D wings as well. There are plenty

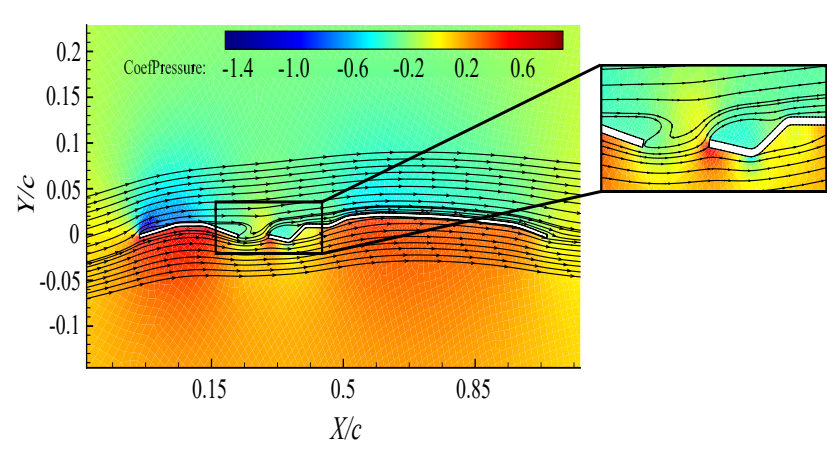

(a)

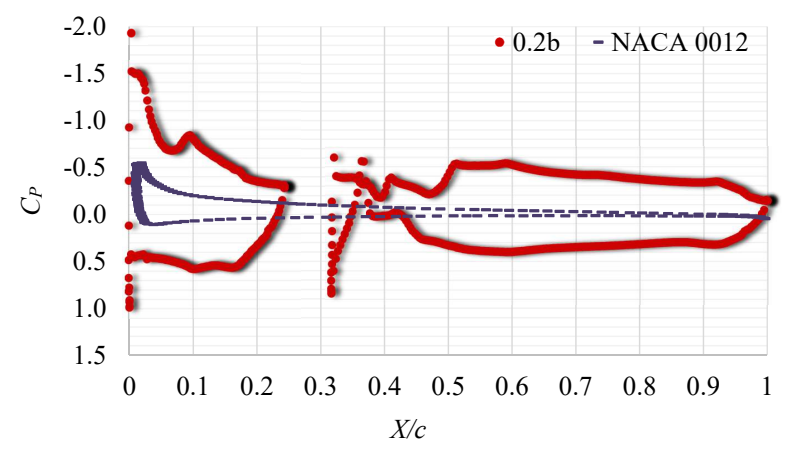

(b)

Fig. 8 Graphical representation of the pressure distribution across chord of a locust-inspired digitized airfoil (0.2b) in a fluid flow at $5^{\circ}$ angle of attack and $R e=7000$; (a) contour of the pressure coefficient with streamtraces indicating flow direction, and (b) quantitative distribution of the pressure coefficient over the chord of our bio-inspired $0.2 \mathrm{~b}$ versus NACA0012 airfoil.

of geometry related factors that influence the pressure distribution over a wing, namely; camber, thickness, aspect ratio, chord etc. To identify each of these parameters' significance and level of influence, we plot the $C_{P}$ distribution over the wing chord quantitatively as shown in Figure 8 (b). This plot indicates the specific-influence of each region on the fluid flow around our airfoil geometry. These regions are classified as local and global depending on their extent of influence, namely; (a) fore and hindwing leading-edge and trailing-edge angle of incidence, strongly affecting the flow separation, upwash, and reattachment of the corrugations slopes. Next, (b) wing-tip being the first point of contact with the flow is a pivotal geometrical feature that must be rounded in contrast to the literature [46]), to achieve maximum structured grids in this region. Furthermore, (c) trailing-edge of the hindwing is of even greater significance due to its

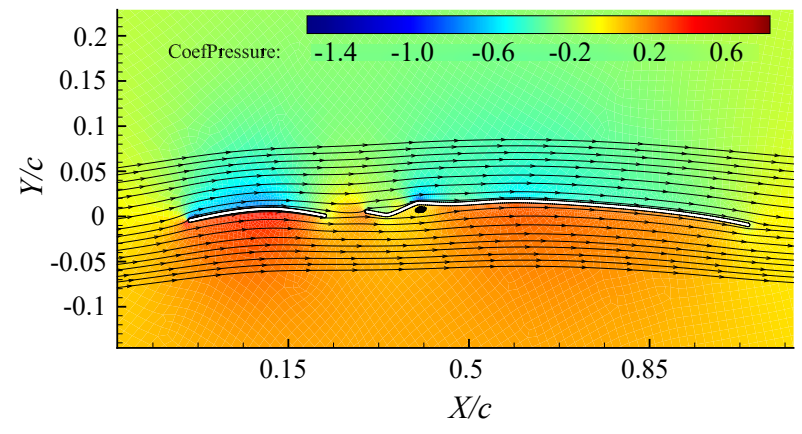

(a)

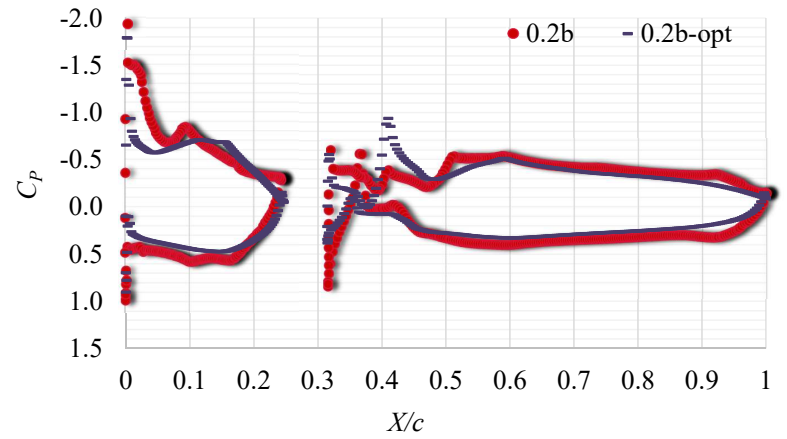

(b)

Fig. 9 Graphical representation of the pressure distribution across chord of a locust-inspired optimized airfoil (0.2b-opt) in a fluid flow at $2^{\circ}$ angle of attack and $R e=7000$; (a) contour of the pressure coefficient with streamtraces indicating flow direction, and (b) quantitative distribution of the pressure coefficient over the chord of our bio-inspired $0.2 \mathrm{~b}$ versus its optimized counterpart $0.2 \mathrm{~b}$-opt airfoil. 
responsibility in generating a downwash that is directly associated with the overall generated lift. And if this section is optimized effectively, lift force can be elevated without compromising drag similar to the conventional high lift devices. Lastly, (d) fore- hindwing phase angle $(\psi)$ with the most significant role in deciding the fore- hindwing interactions, is a crucial energy-recovery parameter that can be exploited in different configurations to further optimize the wing's overall performance. All of the above influential regions and contributing factors are accounted for during the optimization process. From Figure $9 \mathrm{a}$ a), the path of streamtraces indicate that the flow around our optimized airfoil is more laminar and the pressure distribution is more smoothly spread across the wing chord. This improvement is mainly credited to the profiling of the wing corrugations and experimenting with the most optimum fore- hindwing phase shift angle $\left(\psi=4^{\circ}\right)$.

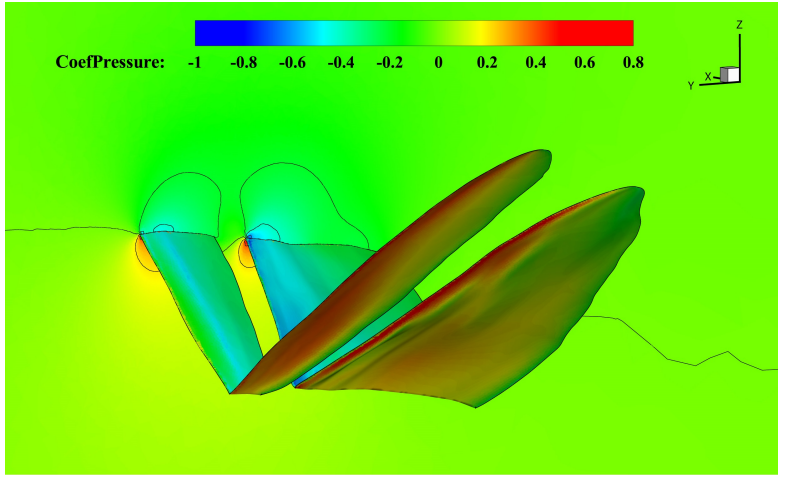

(a)

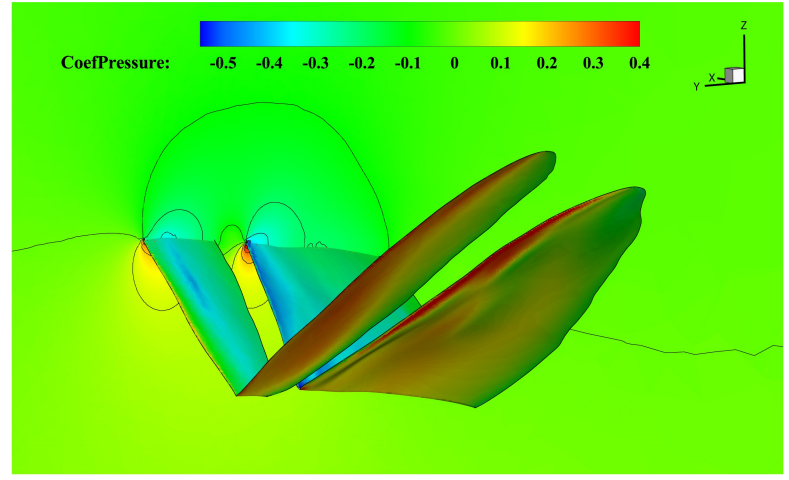

(b)

Fig. 10 Graphical representation of the pressure coefficient contour across chord of the locust-inspired 3D wing in a fluid flow at $5^{\circ}$ angle of attack and $R e=7000$; (a) original (unoptimized) wing, and (b) Nash-GA optimized wing.

To visualize the 3D pressure coefficients for the digitized locust wings, we follow Baker's determination of this insect's gliding posture [61] as described previously. Figure [10 illustrates the $C_{P}$ contour for the (a) original (unoptimized), and (b) optimized locust-inspired digitized wings in gliding mode. The elevated contour lines seen in 10 (b) proves a more distinct pressure difference between the upper and the lower surface of the wing when optimized. Also, comparing the lower surface of the wings qualitatively in the figures, the optimized version demonstrates a more uniform pressure distribution across the wingspan due to the subtle profiling of its corrugations as a result of $25 \%$ PARSEC reduction.

\section{Vortex Topologies}

Here, the original as well as the optimized locust-inspired airfoils and wings are subjected to an incompressible fluid (air) flow at $0.6 \mathrm{~ms}^{-1}(R e=7000)$ that is viscous and transient in nature. The 2- and 3D viscosity for the vorticity analysis is defined by the four equation $k-\omega$-based shear-stress transport (SST) turbulence model [62], and the Spalart-Allmaras Delayed Detached Eddy Simulation (SA-DDES) model, respectively. A low-Reynolds number correction $\left(\alpha^{*}\right)$ that dampens the eddy viscosity, is introduced to facilitate execution of simulations fully-turbulent 
accommodating a transitional Reynolds number regime. Here, the eddy viscosity is defined by a six-equation Reynolds Stress Model (RSM) that is reportedly the most complete physical representation of turbulent flows with a superior ability to simulate and resolve flow separation and reattachments for low $R e$ flows.

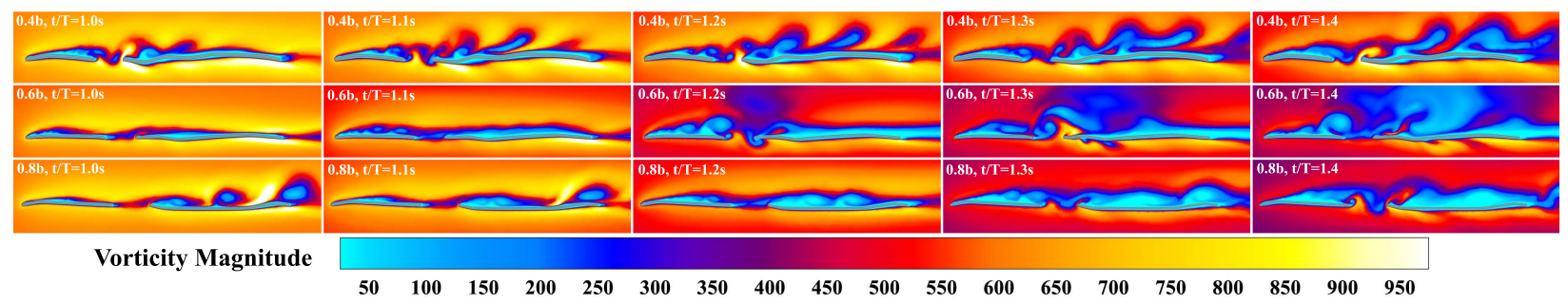

Fig. 11 Illustration of the vorticity contours for the locust-inspired digitized airfoils at $40 \%, 60 \%$, and $80 \%$ spanwise subjected to a fluid flow at $5^{\circ}$ angle of attack and $R e=7000$, sequentially displayed for timestamps 1.0 to 1.4s. Adapted with permission from [50].

Figure 11 graphically demonstrates the vorticity contours of different locust airfoils for five time-steps from left to right starting at $\mathrm{t} / \mathrm{T}=1.0 \mathrm{~s}$ and ending at $\mathrm{t} / \mathrm{T}=1.4 \mathrm{~s}$. If we consider a zero fore-hindfoil phase difference $\left(\psi=0^{\circ}\right)$ for the unoptimized airfoil, two vortical interactions between the trailing-edge of the forefoil and leading-edge of the hindfoil is formed and described as;

1) Leading-Edge Vortices (LEV): Depending on the hindfoil angle of attack, either a separation or a suction region is formed within the gap between fore- and hindfoil that is a result of the passing LEV of the forefoil. These interactions are negligible due to the assumption of zero fore- hindfoil phase difference. However, should a major suction or separation be induced, it may lead to an abrupt acceleration or stall, respectively.

2) The forefoil's passing Trailing-Edge Vortices (TEV) on the other hand, interacts with the hindfoil's leading-edge to create mostly an adverse effect on the thrust. Strong TEV shedding leads to a major flow separation whose energy must be alternatively harnessed to enhance the aerodynamic performance of the tandem wing as a whole. From the above conclusions, it can be inferred that the fore-hindfoil phase difference $(\psi)$ and the TE/LE angles of attack significantly influence the process of energy conservation and restoration which are fundamental in achieving endured flights. For tandem wing fliers, it is common to implement such parameter adjustments for achieving improved aerodynamic performances [17]. Hence, considering these properties during our Nash-GA optimization process further validates and bolsters the results presented in this study.

Comparing the strategy-1 optimized airfoil at $20 \%$ spanwise (0.2b-opt) versus its unoptimized counterpart $(0.2 \mathrm{~b})$ in Figure 12, it is visually evident that the reduced PARSEC parameter (forewing top crest vertical position) at 75\%, results in the formation of a LEV coupled with an active TEV shedding that in turn induces a flow suction near the hindfoil tip. This directly causes further acceleration and hence greater lift coefficient. Furthermore, it is qualitatively evident that the trailing-edge vortices are almost eliminated for the optimized airfoils with only the uniformly shed leading-edge vortices remaining within the flow field. These are collectively the contributing factors for the massive 


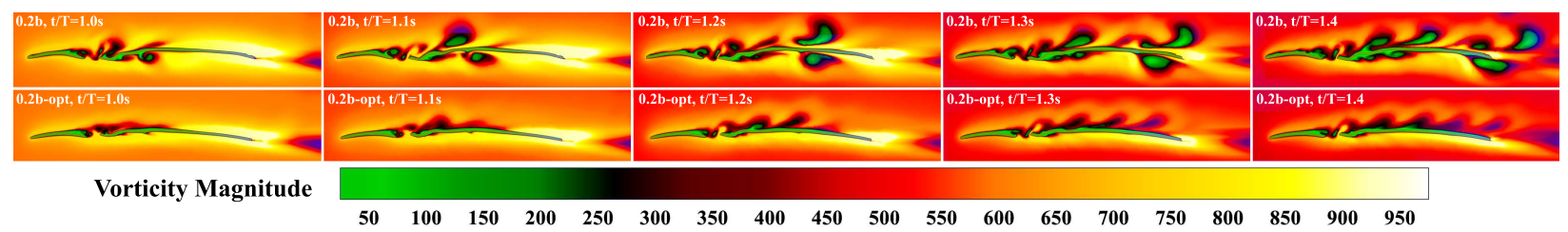

Fig. 12 Illustration of the vorticity contours for the unoptimized $(0.2 b)$ and optimized $(0.2 b-o p t)$ locust-inspired 3D wings subjected to a fluid flow at $5^{\circ}$ angle of attack and $R e=7000$, sequentially displayed for timestamps 1.0 to 1.4s. Adapted with permission from [50].

$(25 \%)$ reduction in drag coefficient of the Nash-GA optimized airfoils.

\section{Digitised locust wings}
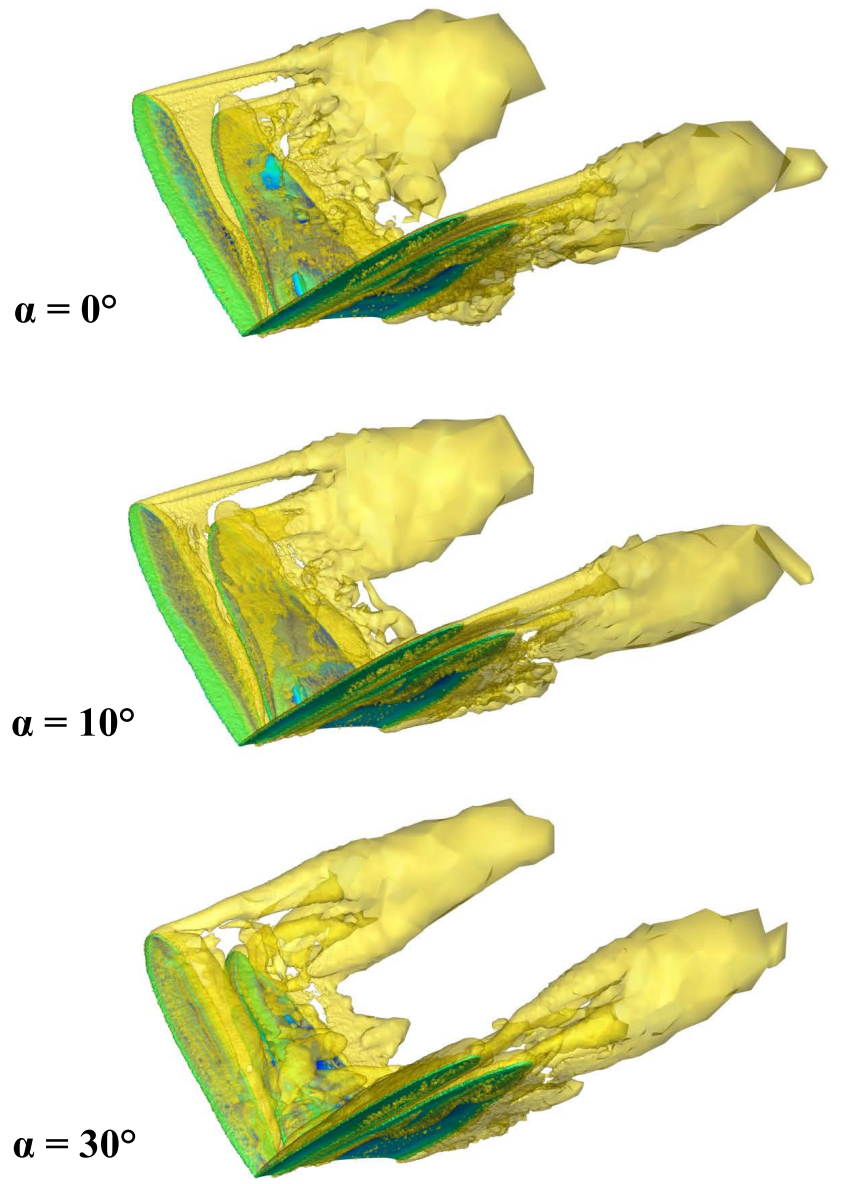

\section{Nash-GA optimised locust wings}
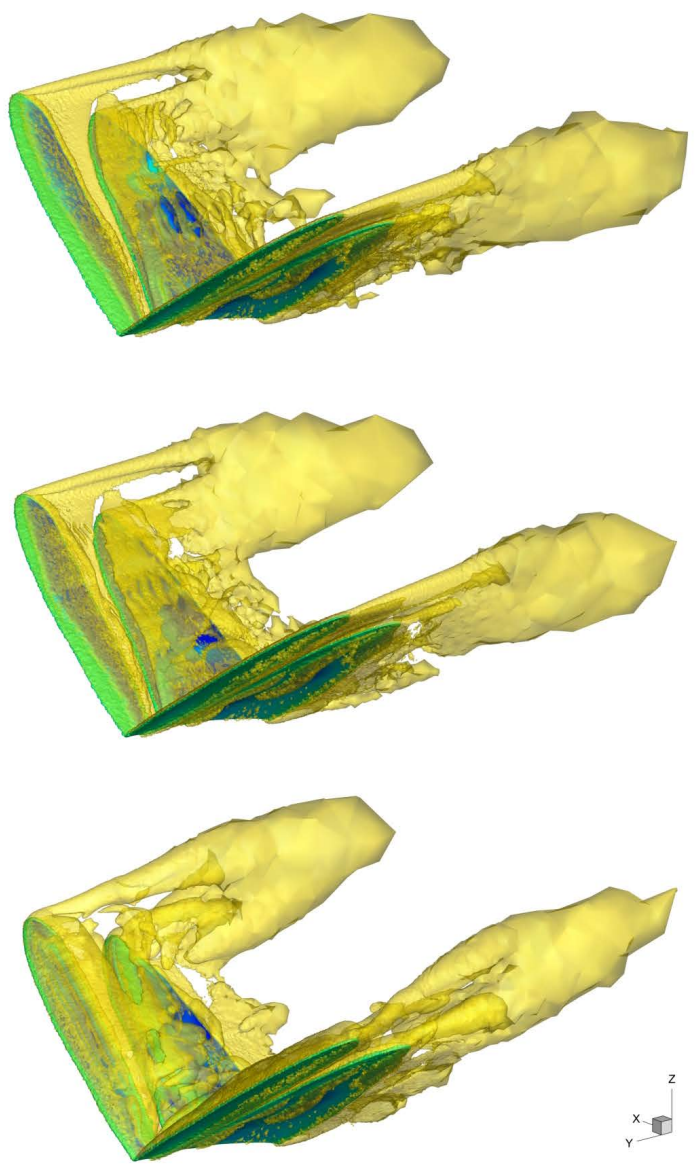

Fig. 13 Graphical representation of the vortex topologies visualized using $Q=3(\vec{U} / l)^{2}[63]$. Left column; digitized locust wings and, right column; Nash-GA optimized wings gliding at three different angles of attack $\alpha=0^{\circ}, 5^{\circ}, 30^{\circ}$ with $\operatorname{Re}=7000$.

Ultimately, the three-dimensional visualization of the wake vortex topologies seen in the Figure 13 is realized using 
Q-criterion [64] that defines vortices as spatial regions where,

$$
Q=\frac{1}{2}\left[|\Omega|^{2}-|\tau|^{2}\right]>0
$$

where $\tau=\frac{1}{2}\left[\nabla v+(\nabla v)^{T}\right]$ is the rate of strain tensor, $\Omega=\frac{1}{2}\left[\nabla v-(\nabla v)^{T}\right]$ is the vorticity tensor, and $\nabla v$ is the velocity gradient tensor. Detailed derivation of the above equations are given in [64].

To represent coherent vortices, positive $Q$ value emphasizes regions of high swirl compared to shear. As explained earlier, the wing orientation is set according to Baker's definition of locust gliding posture [61]. This setup adds $+5^{\circ}$ to the wing's effective angle of attack that is determined by the flow angle of incidence on the wing. It is evident that the flow attachment occurs at lower angles of attack and as $\alpha$ rises flow shedding increases. However, when carefully observed, the optimized wings offer delayed shedding at elevated $\alpha$ which might be due to its slightly streamlined geometry. Also, vortex tubes are formed mainly at the forewing tips that further bolsters the hypothesis of tandem wing configurations being considered as a single sweptback delta wing.

\section{Conclusion}

This study performs a 2- and 3D digital reconstruction of a gliding-expert insect's (locust) wing to unveil the underlying physiological phenomenon responsible for its incredible flight performance. A computational fluid dynamic modeling of the simulated airfoils and wings suggested geometrical features such as corrugations and fore-hindfoil phase angle to be liable for the high aerodynamic efficiencies obtained. Therefore, a novel bio-inspired optimization methodology based on Nash-GA algorithm is proposed to further improve the configuration and geometries of the wing profiles digitized. The process of optimization results in a set of Nash equilibria points facilitating the establishment of a balanced design trade-off strategy hybridized with the conventional energy restoration methods to achieve a satisfactory $77 \%$ improvement of aerodynamic efficiency. However, the optimized airfoils when integrated to form a 3D wing, delivered a 14\% improvement when compared to its unoptimized counterpart. This bolsters the hypothesis suggesting that locust's endured gliding capability emanates from their fore-hindwing and peer-wing vortex interactions which is due to their tandem wing configuration and swarming flight, respectively. Ultimately, future extension of this study [60] focuses on novel fabrication methods to realize the digitized wing designs into prototypes maintaining the presented numerically determined and optimized aerodynamic performance.

\section{Funding Sources}

This work is supported by EU FP7 projects LIVCODE [Grant No.295151]; HAZCEPT [Grant No.318907]; EU Horizon 2020 projects STEP2DYNA [Grant No.691154]; ULTRACEPT [Grant No.778062]; Google Cloud Platform research credits, and partially supported by the National Natural Science Foundation of China [Grant 
No.91648203]; Natural Science Foundation of Hubei Province [Grant No.2018CFB431]; International Science \& Technology Cooperation Program of China [Grant No.2016YFE0113600].

\section{References}

[1] Rayleigh, J. W. S., “The soaring of birds,” Nature, Vol. 27, 1883, pp. 534-535.

[2] Walker, G., “The flapping flight of birds," J. Royal Aeronautical Society, Vol. 29, No. 179, 1925, pp. 590-594.

[3] Ellington, C. P., "The aerodynamics of hovering insect flight. III. Kinematics," Philosophical Transactions of the Royal Society of London. Series B, Biological Sciences, Vol. 305, No. 1122, 1984, pp. 41-78.

[4] Ellington, C. P., “The aerodynamics of hovering insect flight. IV. Aeorodynamic mechanisms," Philosophical Transactions of the Royal Society of London. Series B, Biological Sciences, Vol. 305, No. 1122, 1984, pp. 79-113.

[5] Ellington, C. P., "The aerodynamics of hovering insect flight. VI. Lift and power requirements," Philosophical Transactions of the Royal Society of London. Series B, Biological Sciences, Vol. 305, No. 1122, 1984, pp. 145-181.

[6] Du, G., and Sun, M., “Aerodynamic effects of corrugation and deformation in flapping wings of hovering hoverflies," Journal of Theoretical Biology, Vol. 300, 2012, pp. 19-28. doi:10.1016/j.jtbi.2012.01.010.

[7] Henningsson, P., Hedenström, A., and Bomphrey, R. J., "Efficiency of lift production in flapping and gliding flight of swifts," PLoS ONE, Vol. 9, No. 2, 2014, pp. 1-7. doi:10.1371/journal.pone.0090170.

[8] Hou, D., Yin, Y., Zhong, Z., and Zhao, H., “A new torsion control mechanism induced by blood circulation in dragonfly wings," Bioinspiration and Biomimetics, Vol. 10, No. 1, 2015, pp. 1-10. doi:10.1088/1748-3190/10/1/016020.

[9] Kim, J. K., and Han, J. H., "A multibody approach for 6-DOF flight dynamics and stability analysis of the hawkmoth Manduca sexta," Bioinspiration and Biomimetics, Vol. 9, No. 1, 2014, pp. 1-22. doi:10.1088/1748-3182/9/1/016011.

[10] Koehler, C., Liang, Z., Gaston, Z., Wan, H., and Dong, H., “3D reconstruction and analysis of wing deformation in free-flying dragonflies," J. Experimental Biology, Vol. 215, No. 17, 2012, pp. 3018-3027. doi:10.1242/jeb.069005.

[11] Kesel, A. B., "Aerodynamic characteristics of dragonfly wing sections compared with technical aerofoils," J. Exp. Biology, Vol. 203, 2000, pp. 3125-3135.

[12] Kim, W. K., Ko, J. H., Park, H. C., and Byun, D., "Effects of corrugation of the dragonfly wing on gliding performance," J. Theoretical Biology, Vol. 260, No. 4, 2009, pp. 523-530. doi:10.1016/j.jtbi.2009.07.015.

[13] Meng, X., and Sun, M., “Aerodynamic effects of corrugation in flapping insect wings in forward flight," J. Bionic Engineering, Vol. 8, No. 2, 2011, pp. 140-150. doi:10.1016/S1672-6529(11)60015-2.

[14] Xiang, J., Du, J., Li, D., and Liu, K., “Aerodynamic performance of the locust wing in gliding mode at low Reynolds number,” J. Bionic Engineering, Vol. 13, No. 2, 2016, pp. 249-260. doi:10.1016/S1672-6529(16)60298-6. 
[15] Murphy, J. T., and Hu, H., "An experimental study of a bio-inspired corrugated airfoil for micro air vehicle applications," Experiments in Fluids, Vol. 49, No. 2, 2010, pp. 531-546. doi:10.1007/s00348-010-0826-z.

[16] Luca, M., Mintchev, S., Heitz, G., Noca, F., and Floreano, D., "Bioinspired morphing wings for extended flight envelope and roll control of small drones," Interface Focus, Vol. 7, 2017, pp. 1-11.

[17] Rival, D. E., Hass, G., and Tropea, C., "Recovery of energy from leading- and trailing-edge vortices in tandem-airfoil configurations," J. Aircraft, Vol. 48, No. 1-2, 2011, pp. 203-211. doi:10.2514/1.C031062.

[18] Broering, T. M., and Lian, Y. S., "The effect of phase angle and wing spacing on tandem flapping wings," Acta Mechanica Sinica, Vol. 28, No. 6, 2012, pp. 1557-1571. doi:10.1007/s10409-012-0210-8.

[19] Broering, T. M., Lian, Y., and Henshaw, W., "Numerical investigation of energy extraction in a tandem flapping wing configuration,” AIAA Journal, Vol. 50, No. 11, 2012, pp. 2295-2307. doi:10.2514/1.J051104.

[20] Levy, D. E., and Seifert, A., "Simplified dragonfly airfoil aerodynamics at Reynolds numbers below 8000," J. Physics of Fluids, Vol. 21, No. 7, 2009, p. 071901. doi:10.1063/1.3166867.

[21] Isakhani, H., Aouf, N., Kechagias-Stamatis, O., and Whidborne, J. F., "A furcated visual collision avoidance system for an autonomous micro robot," IEEE Trans. Cognitive and Developmental Systems, Vol. 12, No. 1, 2018, pp. 1-11. doi:10.1109/TCDS.2018.2858742.

[22] Yue, S., and Rind, F. C., "Redundant neural vision systems-competing for collision recognition roles," IEEE Trans. Autonomous Mental Development, Vol. 5, No. 2, 2013, pp. 173-186. doi:10.1109/TAMD.2013.2255050.

[23] Yue, S., and Rind, F. C., "Near range path navigation using LGMD visual neural networks," Proc. Int. Conf. Computer Science and Information Technology, 2009, pp. 105-109. doi:10.1109/ICCSIT.2009.5234439.

[24] Lorenz, M. W., "Migration and trans-Atlantic flight of locusts," J. Quaternary International, Vol. 196, No. 2, 2009 , pp. 4-12. doi:10.1016/j.quaint.2007.09.038.

[25] Weis-Fogh, T., "Biology and physics of locust flight. II. Flight performance of the desert locust (Schistocerca gregaria)," Philosophical Transactions of the Royal Society of London. Series B, Biological Sciences, Vol. 239, No. 667, 1956, pp. 459-510.

[26] Jensen, M., "Biology and physics of locust flight. III. The aerodynamics of locust flight," Philosophical Transactions of the Royal Society of London. Series B, Biological Sciences, Vol. 239, No. 667, 1956, pp. 511-552.

[27] Cloupeau, M., Devillers, J. F., and Devezeaux, D., "Direct measurements of instantaneous lift in desert locust; comparison with Jensen's experiments on detached wings," J. Experimental Biology, Vol. 80, 1979, pp. 1-15.

[28] Shkarayev, S., and Kumar, R., "Instantaneous forces in locust flapping wings," Proc. AIAA Aviation, , No. June, 2014, pp. 1-21.

[29] Shkarayev, S., and Kumar, R., "Kinematics and inertial effects in locust flapping wings," J. Experimental Mechanics, Vol. 56, 2016, pp. 245-258. doi:10.1007/s11340-015-0093-2. 
[30] Simmons, P. J., Rind, F. C., and Santer, R. D., "Escapes with and without preparation: The neuroethology of visual startle in locusts," J. Insect Physiology, Vol. 56, No. 8, 2010, pp. 876-883. doi:10.1016/j.jinsphys.2010.04.015.

[31] Walker, S. M., Thomas, A. L. R., and Taylor, G. K., "Deformable wing kinematics in the desert locust: How and why do camber, twist and topography vary through the stroke?" J. R. Soc. Interface, Vol. 6, No. 38, 2009 , pp. $735-747$. doi:10.1098/rsif.2008.0435.

[32] Kovač, M., Wassim-Hraiz, Fauria, O., Zufferey, J., and Floreano, D., “The EPFL jumpglider: a hybrid jumping and gliding robot with rigid or folding wings," IEEE Int. Conf. Robotics and Biomimetics, 2011, pp. 1503-1508. doi:10.1109/ROBIO.2011. 6181502.

[33] Henningsson, P., Michaelis, D., Nakata, T., Schanz, D., Geisler, R., Schröder, A., and Bomphrey, R. J., "The complex aerodynamic footprint of desert locusts revealed by large-volume tomographic particle image velocimetry," J. R. Soc. Interface, Vol. 12, No. 108, 2015, pp. 1-11. doi:10.1098/rsif.2015.0119.

[34] Henningsson, P., and Bomphrey, R. J., "Time-varying span efficiency through the wingbeat of desert locusts," J. R. Soc. Interface, Vol. 9, No. 71, 2012, pp. 1177-1186. doi:10.1098/rsif.2011.0749.

[35] Le, T. Q., Truong, T. V., Park, S. H., Truong, T. Q., Ko, J. H., Park, H. C., and Byun, D., "Improvement of the aerodynamic performance by wing flexibility and elytra-hind wing interaction of a beetle during forward flight," J. R. Soc. Interface, Vol. 10, No. 85, 2013, pp. 1-15. doi:10.1098/rsif.2013.0312.

[36] Samareh, J. A., “A survey of shape parameterization techniques,” CEAS/AIAA/ICASE/NASA Langely Int. Forum on Aeroelasticity and Structural Dynamics, Williamsburg, VA, 1999, pp. 333-344.

[37] Sobieczky, H., "Geometry generator for CFD and applied aerodynamics," Courses and Lecture International, 1997, pp. $137-158$.

[38] Sobieczky, H., "Parametric airfoils and wings," Notes on Numerical Fluid Mechanics, Vol. 68, Springer, 1998 , pp. 71-88. doi:10.1007/978-3-322-89952-1_4.

[39] Kennedy, J., and Eberhart, R., "Particle swarm optimization," Proc. IEEE Int. Conf. Neural Networks, Vol. 4, 1995, pp. 1942-1948. doi:10.1109/ICNN.1995.488968.

[40] Boggs, P. T., and Tolle, J. W., "Sequential Quadratic Programming,” Acta Numerica, Vol. 4, 1996, pp. 1-52. doi:10.1017/ S0962492900002518.

[41] Goldberg, D. E., Genetic Algorithms in Search, Optimization, and Machine Learning, Addison-Wesley Professional, 1989.

[42] Srinivas, N., and Deb, K., "Multiobjective optimisation using non-dominated sorting in genetic algorithms," Evolutionary Computation, Vol. 2, No. 3, 1994, pp. 221-248.

[43] Nash, J., “Non-Cooperative Games,” Annals of Mathematics, Vol. 54, No. 2, 1951, pp. 286-295. 
[44] Taylor, G. K., and Thomas, A. L. R., "Dynamic flight stability in the desert locust Schistocerca gregaria," J. Experimental Biology, Vol. 206, 2003, pp. 2803-2829. doi:10.1242/jeb.00501.

[45] Kesel, A. B., Philippi, U., and Nachtigall, W., "Biomechanical aspects of the insect wing: An analysis using the finite element method," J. Computers in Biology and Medicine, Vol. 28, No. 4, 1998, pp. 423-437. doi:10.1016/S0010-4825(98)00018-3.

[46] Chen, Y. H., and Skote, M., "Gliding performance of 3-D corrugated dragonfly wing with spanwise variation," J. Fluids and Structures, Vol. 62, 2016, pp. 1-13. doi:10.1016/j.jfluidstructs.2015.12.012.

[47] Anderson, J. D., “Aerodynamics: some introductory thoughts,” Fundamentals of Aerodynamics, McGraw-Hill, 2011, 5 pp. 3-102.

[48] Hu, H., and Tamai, M., "Bioinspired corrugated airfoil at low Reynolds numbers," J. Aircraft, Vol. 45, No. 6, 2008 , pp. 2068-2077. doi:10.2514/1.37173.

[49] Spalart, P., and Allmaras, S., “A one-equation turbulence model for aerodynamic flows,” Recherche Aerospatiale, Vol. 1, 1992, pp. 5-21. doi:10.2514/6.1992-439.

[50] Isakhani, H., Xiong, C., Yue, S., and Chen, W., “A bioinspired airfoil optimization technique using Nash genetic algorithm,” Proc. Int. Conf. on Ubiquitous Robots, Kyoto, Japan, 2020, pp. 506-513.

[51] Della Vecchia, P., Daniele, E., and D’Amato, E., “An airfoil shape optimization technique coupling PARSEC parameterization and evolutionary algorithm,” J. Aerospace Science and Technology, Vol. 32, No. 1, 2014, pp. 103-110. doi:10.1016/j.ast.2013.11.006.

[52] Basar, T., and Olsder, G. J., "Noncooperative finite games: N-person nonzero-sum,” Dynamic noncooperative Game theory, Vol. 23, Academic Press, New York., 1995, pp. 77-160.

[53] Haupt, R. L., and Haupt, S. E., Practical Genetic Algorithms, Wiley-Interscience, 1998.

[54] D’Amato, E., Daniele, E., Mallozzi, L., and Petrone, G., "Equilibrium strategies via GA to Stackelberg Games under multiple follower's best reply," Int. J. Intelligent Systems, Vol. 27, 2012, pp. 74-85. doi:10.1002/int.

[55] D’Amato, E., Daniele, E., Mallozzi, L., Petrone, G., and Tancredi, S., “A hierarchical multimodal hybrid Stackelberg-Nash GA for a leader with multiple followers game," Dynamics of Information Systems: Mathematical Foundations, Vol. 20, Springer, 2012, pp. 267-280. doi:10.1007/978-1-4614-5055-9.

[56] Fudenberg, D., and Tirole, J., Game Theory, The MIT Press, 1991.

[57] Drela, M., and Youngren, H., "XFOIL: An analysis and design system for low Reynolds number airfoils," Mueller T.J. (eds) Low Reynolds Number Aerodynamics. Lecture Notes in Engineering, Springer, Berlin, Heidelberg, 1989 , pp. 1-12. doi:10.1007/978-3-642-84010-4_1.

[58] Deng, J., Zhang, L., Liu, Z., and Mao, X., "Numerical prediction of aerodynamic performance for a flying fish during gliding flight," Bioinspiration \& biomimetics, Vol. 14, No. 4, 2019, pp. 1-13. doi:10.1088/1748-3190/ab23e6. 
[59] Okamoto, M., Yasuka, K., and Azuma, A., “Aerodynamic characteristics of the wings and body of a dragonfly," J. Exp. Biology, Vol. 199, 1996, pp. 281-294.

[60] Isakhani, H., Yue, S., Xiong, C., Chen, W., Sun, X., and Liu, T., "Fabrication and mechanical analysis of bioinspired gliding-optimized wing prototypes for micro aerial vehicles," Proc. IEEE Int. Conf. Advanced Robotics and Mechatronics, Shenzhen, China, 2020, pp. 602-608.

[61] Baker, P. S., and Cooter, R. J., "The natural flight of the migratory locust, locusta migratoria L. II. Gliding,” J. Comp. Physiol., Vol. 131, 1979, pp. 89-94.

[62] Menter, F., Kuntz, M., and Langtry, R. B., “Ten years of industrial experience with the SST turbulence model,” Turbulence, Heat and Mass Transfer, 2003, pp. 625-632. doi:10.4028/www.scientific.net/AMR.576.60.

[63] Suzuki, K., and Yoshino, M., “A trapezoidal wing equivalent to a Janatella leucodesma's wing in terms of aerodynamic performance in the flapping flight of a butterfly model," Bioinspiration \& biomimetics, Vol. 14, 2019, p. 036003.

[64] Jeong, J., and Hussain, F., “On the identification of a vortex,” J. Fluid Mechanics, Vol. 332, No. 1, 1995 , pp. $339-363$. doi:10.1017/S0022112095000462. 